
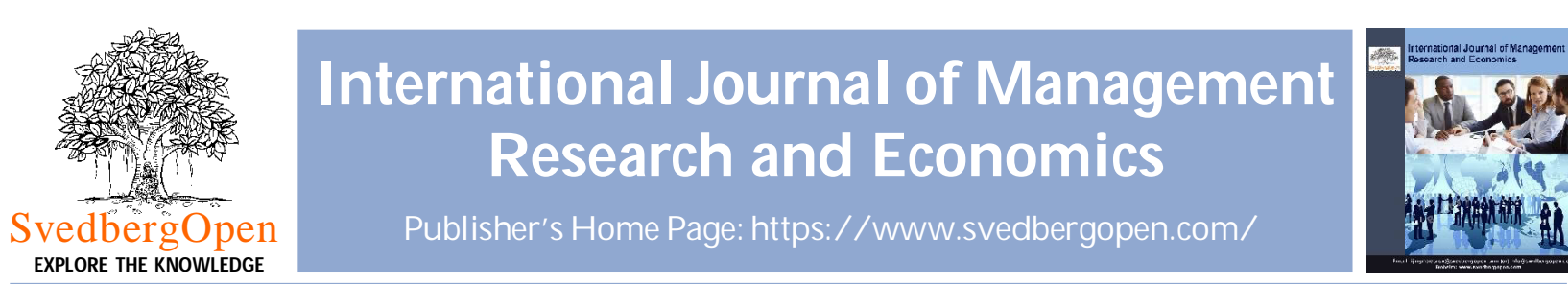

\title{
Corporate liquidity risk management: Coping with Corona and the clearing obligation
}

\author{
Frank Lehrbass $^{1 *}$
}

'Professor for Finance \& Data Science, FOM University of Applied Sciences, Toulouser Allee 53, 40211 Dusseldorf, Germany. E-mail: frank.lehrbass@gmx.de

\section{Article Info}

Volume 1, Issue 1, January 2021

Received : 09 November 2020

Accepted : 15 December 2020

Published : 18 January 2021

doi: 10.51483/IJMRE.1.1.2021.1-26

\begin{abstract}
The European Markets Infrastructure Regulation (EMIR) allows burdening a clearing obligation on non-financial corporations, which formerly did not necessarily clear their business. We give 10 recommendations on how to cope with this obligation. These are motivated by a case study for which we consider a stylized German power producer. For this entity, we derive optimal levels of planned production and forward sales of power using microeconomic theory. Since this results in a significant short position in the German power forward market, we investigate the resulting variation margin call dynamics with a special interest in the ability to forecast worst-case price up moves. We compare different models for the forward log-returns and their performance in $99 \%$ quantile forecasting. A GARCH model with Student-t distribution emerges as the most suitable model. This is used in the case study, which is inspired by data published by the power producer E.ON. Using recent material from the Basel Committee on Banking Supervision we distill the reliable liquidity buffer from an allegedly rich liquidity position and show how suddenly it can be eroded. We point to feedback loops, which make the challenges-posed by the clearing obligation-even more severe. We also spend some thoughts on how to cope with the crisis caused by Corona.
\end{abstract}

Keywords: Industry risk management, Corporate risk management, Liquidity value at risk, Margin at risk, EMIR

(C) 2021 International Journal of Management Research and Economics. This is an open access article

under the CC BY license (https://creativecommons.org/licenses/by/4.0/), which permits unrestricted

use, distribution, and reproduction in any medium, provided you give appropriate credit to the original author(s) and the source, provide a link to the Creative Commons license, and indicate if changes were made.

\section{Introduction ${ }^{1}$}

As a response to the Global Financial Crisis, the G-20 leaders announced in September 2009 that "all standardized OTC derivative contracts should be traded on exchanges or electronic trading platforms, where appropriate, and cleared through central counterparties (=CCP) by end-2012". This decision has led to the Dodd-Frank Act in the US and the European Markets Infrastructure Regulation (EMIR).

\footnotetext{
Parts of this working paper are a revised version of an earlier SSRN Working Paper from May 2013 with the title "Coping with the Clearing Obligation - from the Perspective of an Industrial Corporate with a Focus on Commodity Market". This paper was presented many times, e.g., in 2015 in Frankfurt at a GARP meeting: https://www.xing.com/communities/posts/garp-chaptermeeting-coping-with-the-clearing-obligation-liquidity-concerns-1010023587
}

\footnotetext{
* Corresponding author: Frank Lehrbass, Professor for Finance \& Data Science, FOM University of Applied Sciences, Toulouser Allee 53, 40211 Dusseldorf, Germany. E-mail: frank.lehrbass@gmx.de
} 
Both regulatory initiatives allow burdening a clearing obligation on non-financial counterparties, which formerly did not necessarily clear their business. Thus, a corporation may be put under this obligation if the amount of its nonhedging related derivative business crosses the clearing threshold ${ }^{2}$ in one of five asset classes, which are credit derivatives, equity derivatives, interest rate, foreign exchange, and commodity or others derivative contracts. Thus, it suffices that in only one class the threshold is crossed to put the total derivative business under the clearing obligation. This means that within short term all business has to be margined, including what is called "commercial hedging" 3 .

According to expert judgment (Heemann and Delgado Rodriguez, 2013) 100-150 European non-financial counterparties (here focus on corporations) were expected to come under the clearing obligation. ${ }^{4}$ But even with the most recent EMIRRefit $^{5}$ from 2019 this number still seems very high.

With the clearing obligation the corporation's option ${ }^{6}$ to decide on the well-known "Risk Triangle" is extinguished. No longer can a corporation avoid huge liquidity risks at the cost of bearing some credit risk.

Especially for non-financial corporations (henceforth abbreviated as NFC), which are not systemically relevant, it is remarkable that the risk preference of the "benevolent systemic risk manager", to turn counterparty credit risk into liquidity risk, is now levied on them.

This might lead to surprisingly high increases in liquidity needs as typically commercial hedging is not limited by market risk limits but only the trading operations within a non-financial group. Hence, the existence of market risk limits does not simultaneously limit potentially upcoming liquidity risk. Instead, once commercial hedging has to be margined along with all other derivative business, liquidity risk will become very significant. Another aspect is that market risk is buffered by capital, which might not be sufficiently liquid to cover liquidity risk. Hence, being able to bear market risk does not necessarily mean that one is ready to bear liquidity risk.

It has been argued elsewhere (again Heemann and Delgado Rodriguez, 2013 and also Lehrbass, 2013) that these regulatory developments in the US and Europe lead to a significant increase in concentration risk because it is a relatively small set of banks, which now become even more important than before the Global Financial Crisis through their various roles in and around the central counterparties. Putting the fate of the financial system into the hands of a set of banks runs counter to basic rules of risk management. But here we spell out yet another drawback.

A corporation, which maximizes shareholder value by optimal hedging and by leveraging its expertise via a trading subsidiary, is threatened by the clearing obligation as the corporation does not face a level playing field with the financial industry in terms of access to liquidity. To be more specific we might see a repetition of illiquidity induced nearinsolvencies as in the case of the German company Metallgesellschaft (MG).

MG hedged non-margined forward positions with futures and went close to insolvency due to a liquidity shortfall because although the non-margined forward positions were in the money, the out-of-the-money futures positions led to unsurmountable margin calls. " Ultimately, it was the shortage of cash ... that drove MG to the verge of bankruptcy. Even if the forward contracts generated more gains than the losses from the futures contracts, the negative cash flow from the futures contracts would have subjected MG Risk Management to distress if the losses from the futures were significant enough" (Kartasheva, 2011).

To derive some advice on how to cope with the clearing obligation we go for a dummy case study, which is inspired by some facts about the German power producer E.ON. In this study, we illustrate the potential size of past and potentially upcoming margin calls and discuss how to cope with them - in case a corporation falls under the clearing obligation.

But as this case study needs some groundwork we start first with proving optimal hedging and production decisions ${ }^{8}$ and model a proxy real-world hedging instrument. This allows to derive the liquidity needs due to margining a commercial

2 For each class EUR 3 bn in gross notional value except for equity and credit derivatives, where the threshold is set lower at EUR 1 bn.

3 According to EMIR: Derivative positions taken with an objectively measurable risk reducing effect directly related to the commercial activity, including proxy hedging.

4 There is now also a debate of enforcing margining for non-centrally cleared derivatives. If this comes on top, the issues tackled in this memo will become even more important.

See: https://www.bafin.de/DE/Aufsicht/BoersenMaerkte/Derivate/EMIR/emir_node.html

I.e., to choose the unavoidable two of the following three (angles): market, credit, liquidity risk.

For a short summary see Kartasheva (2011).

This is done to avoid debates about the right hedge ratio as in case of MG. 
hedging position. In the end the reader hopefully can form an opinion how many more MG-like cases we might see due to the regulators" extinguishing the "Triangle" option and treasurers might get an idea of potential decision support models and - at least - some advice for coping with the clearing obligation.

In light of the crisis caused by Corona, we also add some advice from a practitioner's standpoint.

\subsection{Optimal production and hedging}

We give a summary of standard results from microeconomics for intertemporal decisions of a power producer on levels of production and hedging. The time, which is needed to procure the fuels and to build up significant hedging positions in the market place, gives rise to the intertemporal nature of the decision problem. For more details of the relevant microeconomics see Kawai and Zilcha (1986). Here we repeat the crucial results.

Let there be a power producer with future output $x$ in MWh and a stochastic clean dark or spark spread s in units EUR/MWh. Besides, other costs are arising from the power production, which are summed via a non-stochastic, strictly convex, increasing, and differentiable function $c(x) .{ }^{9}$ Power production has to be planned, which introduces uncertainty as concerns the future power and fuel prices. To keep this section easily accessible we apply a two-period model. I.e. instead of considering the full forward term structure we only look at one representative future point in time.

The board of the power producer acts as agent for risk averse stakeholders and therefore maximizes expected utility using a von Neumann-Morgenstern utility function $u(p)$, which—in this case-is a strictly concave, increasing, and differentiable function of uncertain profits $p$.

There is a forward market where the spread can be locked in at today's forward level of $f$. The absolute size of the short forward position is denoted by $z$ in MWh. Hence, the future, uncertain profit from generation and hedging is:

$$
p(x, z)=s x-c(x)+z(f-s)
$$

We denote the expectation operator under the natural measure by $E[$.$] . This allows to summarize the decision$ problem as follows:

$$
\max _{x, z} E[u(p)]
$$

Optimal decisions are derived from the first-order conditions (partial derivatives with respect to $x$ and $z$; denotes optimal levels of decision variables), which are also sufficient conditions for a unique maximum of expected utility. With the Leibniz rule for integrals we get:

$$
\begin{aligned}
& E\left[u^{\prime}\left(p\left(x^{*}, z^{*}\right)\right)\left(s-c^{\prime}\left(x^{*}\right)\right)\right]=0 \\
& \wedge E\left[u^{\prime}\left(p\left(x^{*}, z^{*}\right)\right)(f-s)\right]=0
\end{aligned}
$$

The linearity of the expectation operator allows addition of (2) and (3). Factorization and simplification lead to the following optimality condition:

$$
E\left[u^{\prime}\left(p\left(x^{*}, z^{*}\right)\right)\left(f-c^{\prime}\left(x^{*}\right)\right)\right]=0
$$

Since marginal utility $u$ ' is always positive, the only way to achieve maximum expected utility is by choosing the optimal production level $x^{*}$ such that:

$$
f=c^{\prime}\left(x^{*}\right)
$$

This proves that the output is determined as if the spread were non-stochastic and equal to the forward level. The result is known as "Separation Theorem". For an overview of its history, we refer to Kawai and Zilcha (1986). They spell out the consequences in more detail by stating that the Separation Theorem implies that "any two firms with identical technologies but with different attitudes towards risk and different probability beliefs will produce an equal amount". Hence, the specific degree of aversion towards risk does not play a role in setting the level of future production.

Hence, each power producer plans its level of future production as follows: It looks at the hourly price forward curve $^{10}$ and its share of the stack (i.e., its marginal cost curve $c^{\prime}$ ). It orders the potential future hours of production according to decreasing marginal profit. If the capacity is limited at a level of $\mathrm{h} \mathrm{MWh}$, it chooses the first $h$ hours from

9 Formally we assume c'>0, c" > 0 .

10 Hence, $f$ is not just a single forward price but a vector with a price per hour, e.g., in the next year. Of course not every hour in the year ahead is tradeable. In reality only aggregates are traded. Hence, the reasoning is not applied in hourly granularity but in a coarser manner. 
the ordered list to determine its planned production. Of course in real life, it is impossible to treat each hour on its own and to ignore all other complexities. Burger et al. $(2007,145)$ give the full picture for the interested reader.

From now on we make a practitioner's assumption of unbiased forward markets. I.e., we assume that $E[s]=f$. With this additional assumption, we immediately get a full hedge result due to the following reasoning: The unbiasedness of the forward market enables the power producer to lock in the expected level of profits today by selling an amount of $x^{*}$ at the forward spread. That this is the best decision follows immediately from the definition of risk aversion. ${ }^{11}$ In other words: Locking in the intrinsic value is the optimal hedging decision given the model assumptions.

To indicate the resulting significance for the size of liquidity risk we mention that German power production amounted to-roughly_around TWh 600 in 2012. Let us assume for the sake of simplicity that the sum of $x^{*}$ from all power producers amounts to roughly TWh $600 .{ }^{12}$

Selling the forward spread means going short power and going long the fuels together with carbon certificates. For the sake of exposition, we focus on the power leg. ${ }^{13}$ We can gauge the amount of power sold year ahead for the sake of hedging and have a first look at the liquidity consequences.

Assume now that the producers have sold the planned production fix forward. If power forward prices increase the day after by 1 EUR/MWh the hedging position goes out of the money and leads to a variation margin call of EUR $600 \mathrm{mn}$, which is allocated to the power producers in Germany according to their individual $x^{*}$. This motivates why we are interested in the dynamics of the calendar year ahead contract ${ }^{14}$ and will focus on the probability of forward price up moves. In theory, the margin calls from a short position can go to infinity, whereas the long position has an upper bound. ${ }^{15}$ This underlines why we are striving for a good model to quantify liquidity risk.

\subsection{Modeling the dynamics of a real-world hedging instrument}

We are focusing on the German base power year ahead forward, which is traded OTC. Price data is from the generic year ahead forward time series by Bloomberg. ${ }^{16}$ We assume that the daily log-returns exhibit a similar volatility structure over the past years. We remove the log-returns from the roll dates (31 December to 1 January) and those, which are based on more than five intermediate business days ${ }^{17}$, before we proceed with the estimation of various models.

Of course one could refine the treatment of the rolling by choosing a roll already at the beginning of December as market liquidity shrinks over Christmas for the maturing contract. But the results below show that we already get decent results.

Looking at the in-sample set, which is used for estimation and contains data points from March 2005 to December 2011, we can expect to see the well-known stylized facts of data from financial markets in the log-returns:

1. Financial return distributions are leptokurtotic, that is they have heavier tails and a higher peak than a normal distribution.

2. Skewed returns ....

3. Squared returns have significant autocorrelation, i.e. volatilities of market factors tend to cluster (Engle and Manganelli, 2001).

Therefore we estimate ${ }^{18}$ nine different, "on the shelf" Generalized Auto-Regressive Conditional Heteroskedasticity (GARCH) models as described in Heston and Nandi (1997) and Danielsson (2011).

11 I.e., normally the risk averse entity would even prefer less than the expected profit as long it is certain. Hence, it does not hesitate to lock in profits at the expected level. To see this effect from full hedging one should set $z$ to $x^{*}$ in Equation (1).

12 We will differentiate later on. For now we assume that planned and realized production coincide. The difficult forecastability of green power is certainly one challenge in transfering standard micro-economics to a modern power producer. Other real-world complexities like competitive landscape, dependencies between different plants, common fuel restrictions, technological flexibility etc are ignored as well. Essentially we sketch so-called intrinsic optimization, which accounts for the bulk of the planned production to be hedged.

13 This is also a reasonable assumption when the fuels do not have to be bought in outside markets-as is the case for some lignite stations-or if the fuels are purchased via non-margined contracts.

14 The annual reports of the big utilities in Germany reveal that the forward sale is spread over more than the year ahead. We neglect this detail for the sake of exposition and note that the other forwards (2 years ahead etc.) are somewhat correlated with the year ahead forward.

15 In principle the bound is the nominal value of the position, which is reached once the subsequent forward price drops to zero. But in Germany there are negative prices for power-although so far not often, and not for a whole year.

16 See http://www.bloomberg.com/quote/ELGBYR1:IND.

17 This results from missing data for some days.

18 We use the free of charge software $\mathrm{R}$ and its functions hngarchFit and garchFit. 


\begin{tabular}{|l|c|c|c|}
\hline \multicolumn{2}{|c|}{ Table 1: Comparison of frequencies of exceedance of the 99\% quantile forecasts } \\
\hline & \multicolumn{3}{|c|}{ Exceedance Percentages } \\
\hline Model & HN & ST & GBM \\
\hline In-sample & $1.46 \%$ & $1.16 \%$ & $2.10 \%$ \\
\hline Out-of-sample & $0.38 \%$ & $0.75 \%$ & $1.88 \%$ \\
\hline
\end{tabular}

In his Nobel lecture, Robert Engle gives an intuitively appealing interpretation of the GARCH model: “The GARCH forecast variance is a weighted average of three different variance forecasts. One is a constant variance that corresponds to the long-run average. The second is the forecast that was made in the previous period. The third is the new information that was not available when the previous forecast was made. This could be viewed as a variance forecast based on one period of information. The weights on these three forecasts determine how fast the variance changes with new information and how fast it reverts to its long-run mean" (Engle, 2003b). So basically, we investigate nine ways how to set these weights to get daily variance updates.

It should be noted that the Heston Nandi model is a specialized version of the Heston model, which allows "day ahead" computation of the conditional volatility. This is in contrast to continuous-time stochastic volatility models - like the original Heston model-where the next day's volatility is not known as a function of today's available history of forward prices.

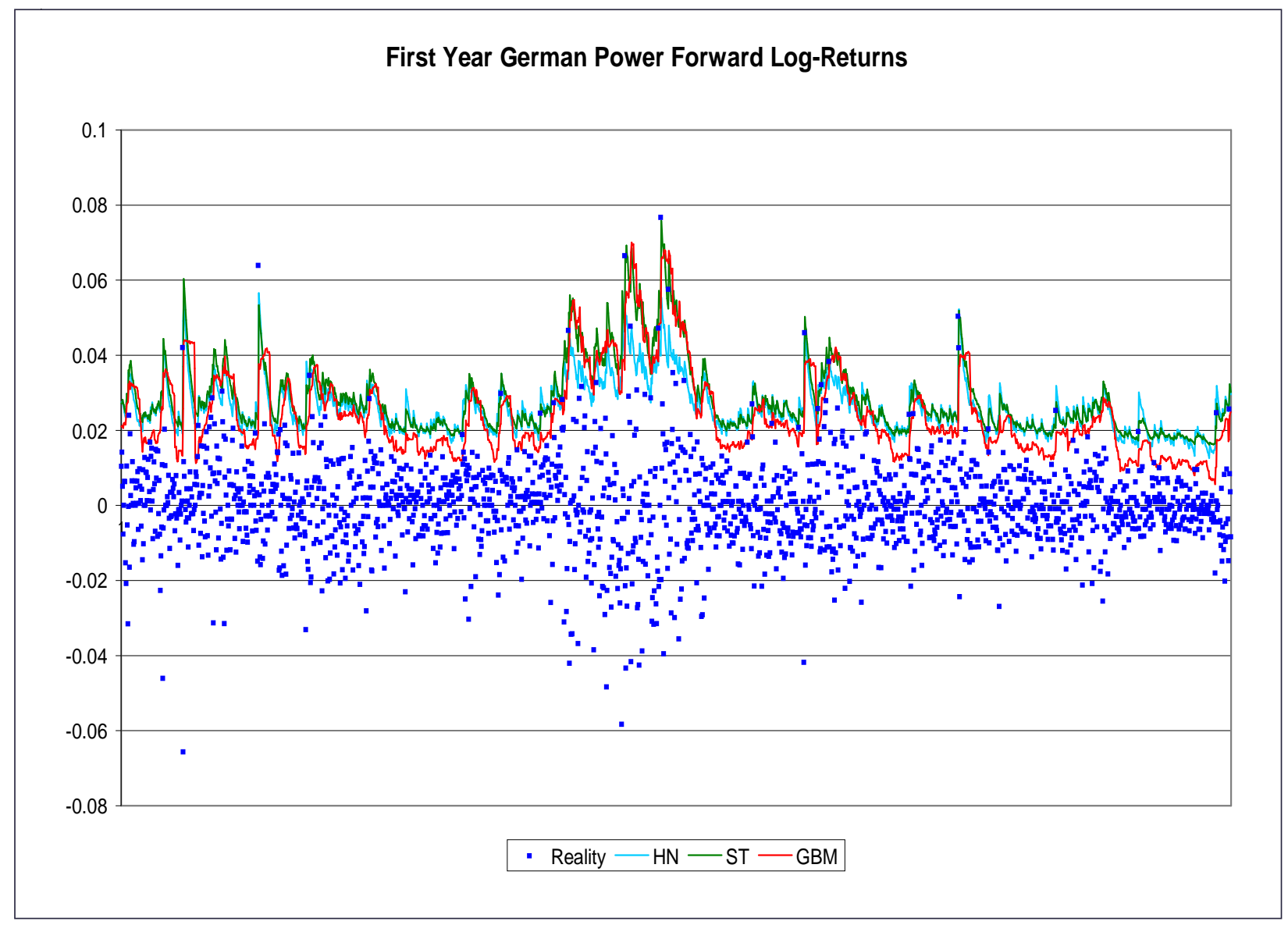

Another important point is that we are working completely under the natural or physical measure and not under the equivalent martingale measure as there is no intent to match option prices, but rather a focus on risk management.

The econometric details are given in Appendix A. Out of the nine GARCH models two are identified as most promising: The Heston Nandi (HN) and the Student-t GARCH (ST). In Appendix A we have checked the forecasting ability of the ST model, which turned out as superior. Of course, every model is wrong — as it simplifies reality-but some are at least useful. This leads to the next step. 
Now we turn to check the reliability of the model as a practical tool to help us setting a liquidity buffer. More important than the in-sample considerations, which are carried out in Appendix A, is the practical usefulness to forecast quantile levels out-of-sample. We imagine that the treasury department of the power producer is interested in "worstcase" up moves of the log-return. I.e., they want to know which level of log-return is exceeded with only $1 \%$ probability.

The sample used for estimation contains 1642 data points (March 2005 to December 2011) and the out-of-sample set has got 266 recordsets (January 2012 up to February 2013).

Table 2: Estimates and their statistics for $\operatorname{GARCH}(1,1)$ Student-t

\begin{tabular}{|l|c|c|c|}
\hline \multicolumn{3}{|c|}{ GARCH(1,1) Student-t } \\
\hline Parameter & Estimate & Standard error & P-value \\
\hline$\omega$ & 0.000004 & 0.000002 & 0.025254 \\
\hline$\alpha$ & 0.086176 & 0.024450 & 0.000425 \\
\hline$\beta$ & 0.889475 & 0.032000 & 0.000000 \\
\hline Shape/df & 6.727741 & 0.983000 & 0.000000 \\
\hline
\end{tabular}

We now compare $99 \%$ quantile forecasts generated by the chosen two models ${ }^{19}$ with a traditional Geometric Brownian Motion (GBM) model, which uses daily updated volatility calculated on the preceding 20 business days. Among practitioners, 20 days is considered to be the minimum sample size for volatility estimation. ${ }^{20}$

The frequencies of the realized log-returns exceeding the $99 \%$ quantile forecast are as follows:

The GBM model underperforms in forecasting the $99 \%$ quantile. ${ }^{21}$ We tested shorter windows for volatility calculation but the results got worse. Ad hoc this is striking as a very short window is close to a conditional variance specification of the following kind ${ }^{22}$ :

$\sigma_{t}^{2}=\ldots \alpha y_{t-1}^{2} \ldots$

But looking at the estimated parameters for both GARCH models reveals that relatively little weight is put on the moving average term. This might partially explain, why the GBM does not have room for improvement by shortening the window length.

On top there remains the simple fact that the Student-t puts more probability into the tails than the normal distribution, i.e., uses a bigger multiplier in forecasting the quantiles.

The ST model performs best in the sense of having exceedances closest to $1 \%$ in the two data sets. The chart shows the in- and out-of-sample results for all three approaches forecasting the $99 \%$ quantile in comparison to realized logreturns (the dots in the chart).

Hence, in the following, we use the ST dynamics for the log-returns of the year ahead forward. As a result of the chosen dynamics, the time-varying variance moves around a long-run mean, which is the unconditional variance, i.e., $\omega /(1-\alpha-\beta){ }^{23}$ Thus, the mean-reverting nature of volatility as in the original model by Heston (1993) or any other established stochastic volatility model is present in our approach.

But this statement should not be overrated. In our set-up, we did not make explicit the specific relation between the dynamics under both measures. Thus it can happen that the mean reversion as generated by the GARCH Student-t under the physical measure vanishes if one moves to the equivalent martingale measure. Since we do not know about the analogous model under the equivalent martingale measure we cannot shed more light on this.

19 We initialize the conditional variance by the unconditional volatility of the ST model, which is $\omega /(1-\alpha-\beta)$. As the HN model gives a zero value for $\omega$, we use the same initialization as in the ST model.

20 We tried a 250 days window as well but the in-sample performance of GBM did not change significantly. Only the out-of-sample performance of GBM improved. However, it would be strange to be convinced by out-of-sample performance only and to forget about the in-sample experience, which is much bigger. On top: The drawback of the bigger window of 250 days is that we loose insample size for backtesting.

${ }_{21}$ Also from the perspective of the supervisory "Traffic Light" or "Three Zone Approach" the GBM does not look too good. For 250 observations 4 exceedances are OK. I.e., $1.6 \%$ is the hurdle, which is crossed in both samples.

22 See Appendix A for the full formalism.

23. See preceding footnote. 
To come full circle with our arguments in favor of the ST model we mention that the estimated ${ }^{24}$ parameters are highly significant. They are made explicit with their statistical Table 2 . The formulae, which define the ST model, are as follows:

Denoting the log-returns of the year ahead forward by we can specify the ST model as:

$Y_{t}=\sigma_{t} Z_{t}$

with a time-varying variance

$\sigma_{t}^{2}=\omega+\alpha y_{t-1}^{2}+\sigma_{t-1}^{2}$

where $z_{t}$ is iid Student-t distributed.

The "Shape" or "degrees of freedom" parameter in the last column is significantly below infinity and underlines that it is worthwhile not to work with a normal GARCH but a Student-t model.

\subsection{Dynamics of variation margin}

If $M(t)$ is the $M t M$ of a position and $Q(t)$ its size (e.g., MWh power), the level of variation margin at the point in time $t$ is the negative of $M(t)$ times $Q(t)$. I.e., if this product is negative, we are out of the money and have to pay variation margin $(V M)$ amounting to the monetary size of the product of $M(t) Q(t)$. Hence, we have:

$-V M=M(t) Q(t)$

For short time intervals, the change of $M(t) Q(t)$ might be derived by first differentiation with respect to time $t$. Application of the product rule gives in usual notation:

$(M(t) Q(t))^{\prime}=M^{\prime} Q(t)+M(t) Q^{\prime}$

The right-hand side of Equation (9) can be read as:

Change in market prices times known positions + known MtM times change in position

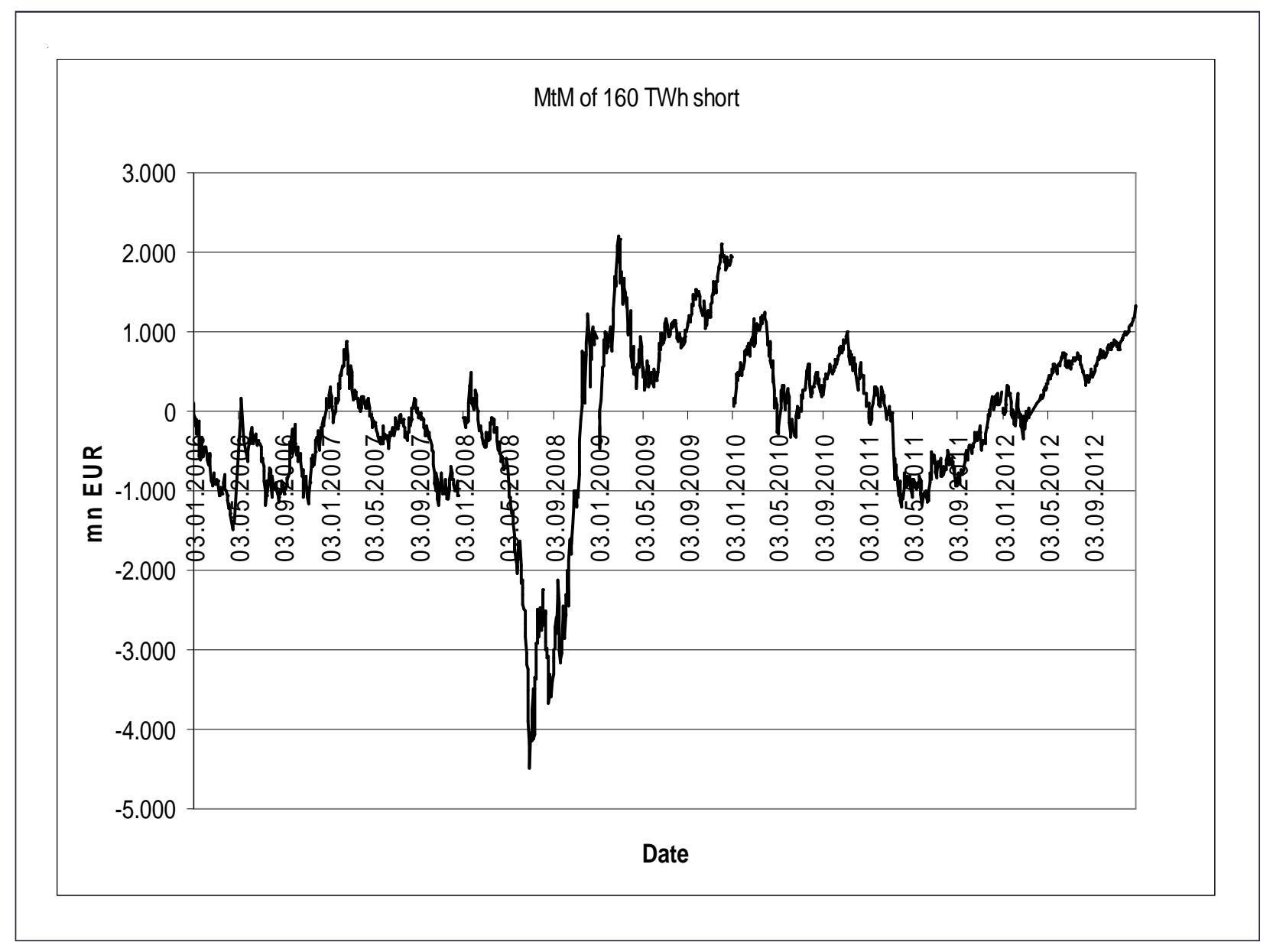

24 Estimation with Maximum Likelihood. 
Assuming unchanged positions the variation margin can be derived for very short-term intervals-like a single day-by multiplication of the existing positions with $M^{\prime}$ '. Assuming for the sake of exposition that the power producer only has got one position, namely $x^{*}$ short in the year ahead forward, we can model the dynamics of the liquidity needs for the German power producers as a whole by scaling up the stochastic process $y_{t}$ with the planned production for the year ahead. Positive values for $y$ mean an up move in the year ahead forward and put the existing full hedge out of the money, which in turn necessitates paying variation margin — given the clearing obligation.

Before we proceed with a worked-out example we note that for longer time horizons, we must look at first differences.

$\Delta(\mathrm{QM})=(\mathrm{M}(\mathrm{t})+\Delta(\mathrm{M}(\mathrm{Q}(\mathrm{t})+\Delta(\mathrm{Q})-\mathrm{M}(t) Q(t)$

Or equivalently:

$$
\Delta(\mathrm{QM})=\mathrm{Q}(\mathrm{t}) \Delta \mathrm{M}+\mathrm{M}(\mathrm{t}) \Delta \mathrm{Q}+\Delta \mathrm{M} \Delta \mathrm{Q}
$$

There is an additional ("mixed") term for longer time intervals to the right side. Hence, for real-world applications with a longer time horizon, we can expect a more complex model.

What has got evident from the preceding discussion is that the hope for a normal distribution is in vain. For short term returns (i.e., $M^{\prime}$ ') Engle and Manganelli (2001) have pointed out the violation of the assumptions underlying the central limit theorem of probability theory. Hence, for a single position considered over longer terms like a month, there is no convergence to normality. This is the time-series perspective.

There is also the cross-sectional view or what one can call "portfolio view": Given a fixed time horizon for risk analysis, the positions to be margined do not generate a collective normal distribution. First, many commodities move in tandem, i.e. are not stochastically independent. Second, as a rule, there will be at least one dominating position like the short power position of a utility. Third, the number of different positions will be far below one hundred. Hence, any result which is based on converging in number to infinity is not readily applicable.

\subsection{Simplified dummy case study inspired by data from the power producer E.ON}

In the following, we try to create a dummy case and take some inspiration from data published by E.ON. In no way do we claim that this is actually what E.ON does, has done, or will do/experience. Especially we do not forecast whether E.ON will fall under the obligation or not. To make it a relevant case study we simply consider a dummy, which has fallen under the obligation and is treated as a bank as concerns liquidity risk handling.

First, we use the available data for the year ahead forward to do a "dummy" ex-post simulation of the MtM of a

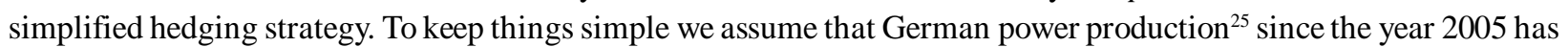
been around TWh 600 p.a. and that E.ON has got a share of a sixth of it.

For the year 2011, we can cross-check the sanity of this assumption using E.ON's published ${ }^{26}$ "Facts and Figures 2012", where they report for the year 2011 a nuclear output of TWh 45.4, gas-fired output 6.2, coal-fired of 34.2, and renewable of 23.9. This sums to TWh 109.7 production in 2011, i.e., roughly TWh 100 . Assuming that only the thermal part of it can be planned in standard micro-economical fashion, we $\operatorname{set}^{27} x^{*}$ per year to TWh 80 , which we assign to our dummy power producer to set the stage.

We want to generate a meaningful time series of variation margin for our dummy. The most useful source to get a basic understanding is E.ON's presentation to debt investors (E.ON 2012). Therein it is stated that per 2012 E.ON had already sold forward 100\% of the production for 2012 and 2013 (year ahead) and $90 \%$ of the production for 2014 (E.ON 2012, p. 39). Hence, we may multiply $x^{*}$ by two.

To avoid modeling the two-year ahead forward we assume for simplicity that we always have a flat forward curve in years one and two. ${ }^{28}$

E.g., we assume that our dummy has determined $x^{*}$ for the year 2007 and 2008 and sold forward two times $x^{*}$ at the beginning of January 2006. Hence, we need to know the first available forward quote of our time series in 2006 and can then mark the short position through $2006 .{ }^{29}$ The result is shown in the next chart:

25 This figure is roughly sensible. See BDEW's chart: http://bdew.de/internet.nsf/id/DE_Energiedaten, “Brutto-Stromverbrauch in Deutschland".

26 See: http://www.eon.com/en/about-us/publications/facts-and-figures.html

27 Ideally one would have E.ON's marginal costs figures for the last years, power forward curves and then apply the Separation Theorem. What we do here is assuming that the realized production has been the ex-ante optimal dispatch.

28 For a storable commodity this could open up arbitrage, but here we talk about non-storable power.

29 The data for 2005 is incomplete. Hence, we start in 2006. 
The MtM of the hedging position is quite volatile, which is no surprise in light of the preceding discussion of the dynamics of the (representative) hedging instrument. The gaps in the curve are due to closing the position at year-end and opening a new one on the first business day of January. Of course, this is highly artificial as our dummy would never do all hedges in one day for reasons of market liquidity, but we get the big key insight ${ }^{30}$ :

The primary lesson of the above chart is the potential size of the negative MtM. In the past, the negative MtM led to a margin call only for those positions, which were done under a margining agreement. In light of the size of the EEX, it is clear that part of it had to be done OTC. Which part was chosen by E.ON to be margined is not publicly evident. For the non-margined positions, one can guess that E.ON's counterparties from time to time had to be willing creditors to E.ON.

As concerns our dummy we can expect that if the already granted credit is big enough, our dummy will certainly find correctly incentivized counterparties to give more credit for the non-margined business.

But if our dummy falls under EMIR's clearing obligation, it will get more difficult to avoid sudden margin calls of significant size in the future-while maintaining the optimal hedging strategy. In our dummy example within a short period of time in the year 2008, the MtM dropped from near zero to minus EUR $4 \mathrm{bn}$.

So the natural question arises whether this can repeat itself. In light of the political influence on power markets, such sudden moves cannot be precluded. This ensures that things will not calm down and hedging is worthwhile to cope with power price uncertainty.

Let us assume that in our dummy example TWh 160 were sold fix on the first business day of January 2013 at 45.15 EUR/MWh base. To get a first impression of potential cash needs assume an immediate move of the German base forward to $46 \mathrm{EUR} / \mathrm{MWh}$. By this, we get a negative MtM of EUR $136 \mathrm{mn}$.

However, it must be noted that we are focusing on the power leg solely. Taking into account the fuel hedge positions might change the picture, but also render it more complicated as the market liquidity is different from the power markets. To come back to our main message: The problem with the clearing obligation is the MG-like situation of our dummy power producer.

Now we ask how our dummy can cope with sudden margin calls - assuming that it applies bank-like risk management thinking. From a treasury perspective the reactive measures might be pondered in the following order:

1. Cash flow from operations

2. Issuance of Commercial Paper (CP)

3. Usage of uncommitted lines

4. Usage of committed lines

As concerns the latter two items the Basel Committee on Banking Supervision ${ }^{31}$ (Basel, 2013) expressed some doubt. With respect to the real-life availability of liquidity lines committed by banks, it is stated: "that .. banks may not be in a position to honor credit facilities, or may decide to incur the legal and reputational risk involved in not honoring the commitment, to conserve their own liquidity or reduce their exposure".

Looking at the second measure one might apply similar doubts as the CP market might be inaccessible when needed most. Besides the ability to issue $\mathrm{CP}$ at attractive levels is dependent on back-up liquidity lines. Hence, any $\mathrm{CP}$ program must be seen in conjunction with the availability of committed lines.

Considering the measure number one it is important to be aware that power producers are investing a lot. Hence, it counts what is left over from the operating cash flow (e.g., E.ON reports for the first nine months of 2012 an operating cash flow of EUR $6.8 \mathrm{bn}$ vs investments of EUR $4.3 \mathrm{bn}$ ). On top, there are contingent obligations from project development activities. A good example is the rumors ${ }^{32}$ about the Brazilian billionaire Mr. Eike Batista, who ponders-partiallywithdrawing from the joint venture with E.ON in Brazil. Thus a sudden need for cash to buy shares of the joint venture might arise. Taking such contingent claims into consideration makes it advisable to look at the available liquidity buffer, which is under full control of E.ON, to motivate a sensible buffer size for our dummy case.

30 Some might immediately ask: But what about the other forward positions? Doesn't it even out somehow? It depends on whether one hedges the other legs of the dark, spark ... spread in margined manner. A portfolio consideration is left for future work. E.g., in a "GARCH-copula approach" the various GARCH processes would be tied together with a suitable copula (Pfaff, 2013, 145).

31 There is an earlier paper by the BCBS from 2008, which can be used as additional food for thought.

32 E.g. as reported in Borsenzeitung as of 14 March 2013, page 8. As of 18 March there are rumours of E.ON investing around EUR 700 into MPX: By this they would increase their share in MPX to around 40\% (Source Suddeutsche Zeitung, page 22). 
E.ON's informs on a "strong liquidity position" of EUR 13.4 bn of liquid funds and non-current securities and an undrawn EUR 6.0 bn revolving credit facility. In light of Basel's doubts, we do not consider the facility. In addition to the view of Basel (2013), we mention that one should not underrate the negative signaling implied by drawing.

Instead, we focus on liquid funds \& non-current securities. The latter might be pledged and thereby do not form liquid assets under the control of E.ON. This leaves us with the liquid funds of-we guess according to E.ON's annual report 2011—presumably EUR 7 bn.

As E.ON does not only hedge and trade in commodities, this buffer has to be shared with what might come from the foreign exchange and interest rate derivatives. Again we approximate with the use of E.ON's annual report. The reported total volume of non-commodity derivatives is EUR 47 bn and of commodity-related ones EUR 199 bn, whereof power forwards sum to roughly EUR $88 \mathrm{bn}$.

Hence, for our dummy, we assume that only a fraction of $36 \%(=88 / 246)$ of the buffer is available for margining the power forward position, i.e., EUR $2.52 \mathrm{bn}$. A true portfolio approach could lead to a much more benign picture, e.g., if all prices moved upwards simultaneously E.ON would benefit from its long positions in fuels and receive margin. ${ }^{33}$

To underline the dummy nature of our considerations we henceforth work with a rounded figure of EUR 2.5 bn for the size of the dummy's liquidity buffer.

Now we investigate, which margin calls might arrive at our dummy in 2013, where TWh 160 were sold fix on the first business day of January at 45.15 EUR/MWh. We are interested in the probability that EUR 2.5 bn turns out to be insufficient. Basic arithmetics give the result that the forward has to move up to $60.78 \mathrm{EUR} / \mathrm{MWh}$, which corresponds to a $\log$-return of $30 \%$.

Of course, this is a rare event that such an up move occurs in a single day. Therefore we run 200 simulations ${ }^{34}$, each for 250 trading days, using our GARCH(1,1) Student-t model and check in how many runs the resulting variation margin (negative of MtM) due to a TWh 160 short position exceeds EUR 2.5 bn. This happens in $4.5 \%$ of the 200 runs. This must not be understood as a default probability as there might be other sources of liquidity like the lines granted by banks. But as said it might be that their availability is not as expected.

The set of simulations is in no way representative. To judge on the first passage / hurdle crossing probability one must do more runs. As we do not try to forecast this probability here, we do not elaborate on the simulation.

Of course, this investigation implicitly assumes that our dummy does not react to increasing forward prices orsynonymously_rising losses in its hedge position. But in real life, any reaction means locking in losses, which diminishes the size of the remaining liquidity buffer. Maintaining the desired power hedge implies in real life that the hedge is transferred to an un-margined territory, e.g., changing from EEX to mid-sized corporate counterparties. Thereby liquidity risk is transferred back to credit risk. The impact of EMIR is that this option can be significantly reduced in scope.

On top, the dummy can also try to raise additional cash if the buffer is exhausted. This is what MG tried and it is discussed as follows: "Managers can access capital markets by either issuing bonds or equities. Alternatively, they can borrow from banks. However, there is a risk that, when the manager goes out to the market to secure funds to settle the losses from futures positions, the market will view the company as being distressed. Losses from derivatives trades have a very negative connotation and the market would certainly react negatively toward the news of its losses and the manager's effort to secure funds, potentially increasing the funding cost significantly. Ideally, the manager can use the forward positions, which have been generating gains, as collaterals to mitigate the negative perception, claiming that the company has valuable assets on its balance sheet in the form of contracts with customers that will generate positive cash flows going forward. However, convincing the market would still be a difficult task. [...] Ultimately, it was the shortage of cash - not necessarily the losses themselves - that drove MG to the verge of bankruptcy" (Kartasheva, 2011).

Naturally, we could extend this dummy case study from margin or liquidity at risk considerations to a full-blown liquidity value at risk measurement by including the potentially increasing funding costs, etc. In such an analysis the full P\&L impact could be investigated and a worst-case impact would be quantified by some quantile. Hence, additional value drivers like increasing funding costs would be considered and ideally differentiated according to funding instruments, etc. This again would call for a full term structure model of funding costs in combination with detailed

33 This in turn assumes constant dark spreads. Hence, it is worthwhile to have a look at the spread dynamics in more detail. This will be reserved for future work.

34 Doing more than 200 runs overcrowds the chart. 
modeling of the liability side of the corporation. For the sake of brevity, we do not tackle this here and conclude the dummy case study with a short glimpse at the involved potential feedback loops. We give a rough illustration based on our understanding of E.ON's published data.

First, we note that posting of cash increases the "debt factor" (DF) of E.ON (E.ON 2012, p. 6). We only look at its level in case EUR 2.5 bn of variation margin has been posted. As of Dec 2011, the DF is:

$\mathrm{DF}_{2011}=$ Economic net debt / EBITDA $=3.4$

In light of Economic net debt (END) of EUR $36.385 \mathrm{mn}$, this implies an EBITDA of EUR $10702 \mathrm{mn}$. Posting EUR $2.5 \mathrm{bn}$ of variation margin increases END by the same amount and brings the DF to a level of roughly 3.6 (due to less cash ${ }^{35}$ ).

Second, we remark that any increase in the DF puts pressure on the rating of E.ON. In case a downgrade to BBBoccurs we can expect that threshold levels in margining agreements go to zero, i.e., credit is withdrawn from E.ON. By the same amount cash has to be raised and increases END further (due to increased debt).

Third, in case volatility in the power markets increases, e.g., by entering a volatility cluster, initial margins certainly go up and, again, cash has to be raised.

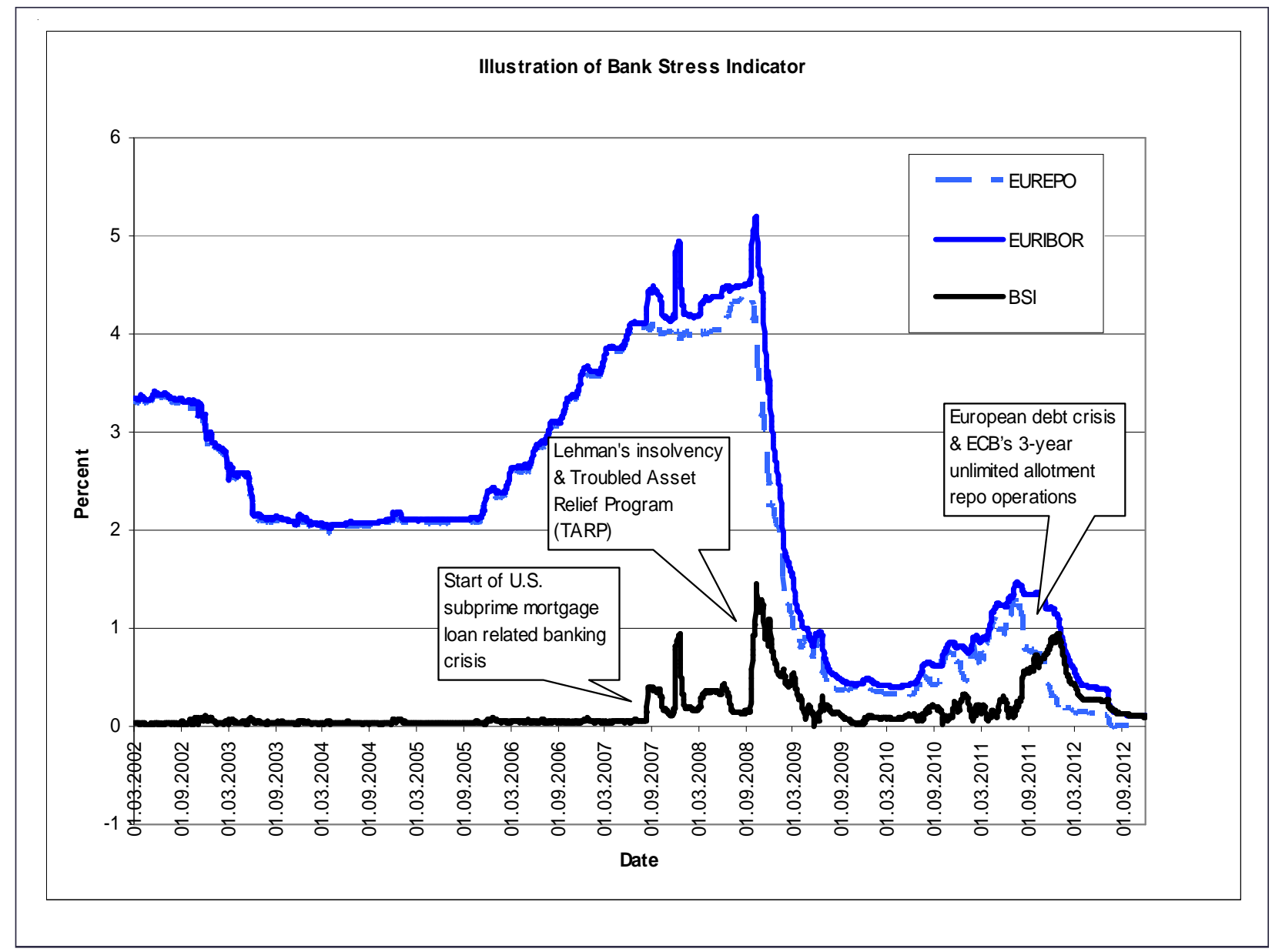

Fourth, there might also be an impact on independent amounts, which might go up. Normally a well-rated counterparty would not be subject to an independent amount, which is the analog on to the initial margin in OTC style margining. But with EMIR there is a push towards central clearing and, hence, a transformation towards obligatory initial margins. The effects on the DF are clear.

The important take away is that a corporation might enter an adverse feedback loop: The more cash it needs, the less creditworthy it gets ceteris paribus.

Note what is going on here: A corporation has hedged and thereby stabilized the value of the firm. But the rating might be strongly driven by the DF and too little consideration might be given to the improved value of the natural power long position!

$35 \quad$ I.e., $36.4+2.5=38.9$ divided by 10.7 . 
As said a fully-fledged model is out of scope here, but the message has got evident: EMIR can push a non-financial corporation into dangerous waters. Treating a corporation like a bank—without granting a level playing field—can peril the corporation's health. More on this theme follows in the next section.

\section{Conclusion - 10 recommendations}

1. Choose a dynamical model, which performs well under the real-world measure, for forecasting margin at risk and strive for a fully-fledged liquidity value at risk model

First, we have seen that relying on too simple dynamics can lead to endangering situations. Table 1 has shown that even with updating the volatility of a Geometric Brownian model on a running basis this model does not serve well in forecasting the $1 \%$ worst case margin at risk. Large price up moves of the hedging instrument occurred roughly twice as often. Thus anyone who uses the GBM model for determination of a sufficient liquidity buffer might experience a shortage in liquidity more often than planned. In contrast, the GARCH model with the Student- $t$ distribution delivered what it promised. The $99 \%$ quantile was exceeded with roughly $1 \%$ probability in- and out-ofsample.

Second, we have had a glimpse at the potentially fatal feedback loops, which makes consideration of the interdependencies of liquidity and (own) credit risk worthwhile.

2. Have an eye on implied volatilities and political events, because it might be time to recalibrate your model

Korn et al. (2011) recently pointed out that implied volatilities contain superior information for risk management purposes. In retrospect, it has proven beneficial to move from backward-looking historical volatilities to forwardlooking ones in times of stress.

This advice certainly applies to the GBM model, which has been considered above. The useful property of the GARCH models is that they incorporate volatility clustering.

But using a GARCH model does not replace a holistic view on your key risk drivers. E.g. on 16 April 2013 the European Parliament voted down a proposal to make it more expensive for companies to burn fossil fuels. Such events should be on the radar screen of the liquidity risk managers in addition to useful models.

3. Have an eye on costs of collateral for interbank lending, because it indicates situations in which your house bank might not be as reliable as you thought

But even with a good model for liquidity planning, one might see surprises as they have been spelled out recently by the Basel Committee on Banking Supervision (Basel, 2013).

Whether banks are in stressed conditions is often indicated by: "the spread between unsecured and secured loans in the interbank market" (de Socio, 2011). As the overnight interbank market is sometimes driven by technicalities, we use as an example indicator the difference between the one month Eurepo and Euribor. The above chart ${ }^{36}$ shows that our indicator's spikes are linked to real-life crisis events. On the other hand, the indicator goes back to normal as soon as rescue measures by central banks and governments are put into place.

But the indicator does not only serve well to signal systemic crises. Effectively it shows how the costs of repo collateral develop because the difference between the two rates is exactly this by virtue of arbitrage arguments. ${ }^{37}$ Thus, the indicator also helps to monitor a presumably upcoming "collateral squeeze" 38 and could be a helpful input for collateral management.

On top, it might also indicate the effective willingness of banks to honor their obligations under committed liquidity lines.

4. Make sure your house banks (want to) understand your business

As the case of MG has shown a bank, especially if itself under stress or striving for other goals, might start doubting the solvency of the power producer, which is asking for liquidity at short notice.

On top, like in the case of MG there might be no desire or ability to interpret the margined position as a hedge for a non-margined position, which is significantly in the money. Thus the effectively constant value of the power-

36 We use the maximum available time series. For more details see: http://www.euribor-ebf.eu/. As we do not estimate a dynamical model for this spread, we do not split the data into in-sample and out-of-sample.

37 E.g., if Euribor is bigger than Eurepo + cost of collateral, raise cash in secured format and offer it at Euribor to lock in the arbitrage gain. Or if Euribor is below Eurepo, raise cash in uncollateralized format and lend it at Eurepo and get collateral.

38 Of course, the squeeze can be softened by the ECB as has been done through lowering the eligibility criteria. But in the meantime problems can get big. 
producing firm (due to being hedged) might be put into question with the result that lines are not honored or new ones are not granted.

If the bank is a bit too open in expressing its doubts in the media (e.g., Deutsche Bank on Leo Kirch's group ${ }^{39}$ ), the liquidity situation of the power producer might come under additional pressure due to lines being canceled and general credit withdrawn. A downward spiral like this could be seen many times in history. The ability of a power producer to manage the entailed reputational risk is rather limited—especially at short notice.

For NFC with an attractive asset base like in oil and gas exploration and production (E\&P) three-way agreements might be a solution to preempt MG like situations: "In one common structure, E\&P firms enter into a three-way agreement with a hedge provider — typically a physical player, such as BP, Shell Trading or Cargill—and a bank. The bank agrees to provide the E\&P firm with credit to support its hedge, secured against the firm's oil and gas assets. Meanwhile, the bank and the hedge provider sign an inter-creditor agreement specifying how the two of them will divide up their claims against the company's assets in the event of a default. The advantage of such a structure, say market participants, is that eliminates the risk of cash margin calls" (Osipovich, 2012) and that they can be set up in advance.

For NFC, which lack attractive and yet unpledged assets or face unwilling banks and/or hedge providers, there is no alternative to having a suitable liquidity buffer at hand.

5. Watch out for "hostile" entities, which have understood your business

If the market place knows that an entity is hedging (e.g., an airline hedges its natural short kerosene position by going long forward), hedge funds or other smart traders might try to push the hedging position out of the money. Thus, they can increase the insolvency probability ceteris paribus - thanks to the clearing obligation.

6. Be aware that you are regulated nearly like a bank, but do not face a level playing field

There is a serious disadvantage in comparison to banks. A bank always can resort to the central bank if its liquidity situation is endangered. For a NFC this door is locked. In the old days before the clearing obligation, there was always the alternative to seek credit from the counterparty (e.g., stop bilateral margining). This door has been locked now.

Of course the NFC could set up a bank. But this bank would have to be of significant size to be able to provide sufficient liquidity due to regulatory limits on large loans.

7. Make up your mind on the right mix of hedging counterparties in due course

The only way for the NFC to escape the clearing obligation is to stop hedging with counterparties, which have fallen under the clearing obligation, too.

There are two sets of counterparties, where things stay the same after EMIR: Business with banks (e.g. to access the $\mathrm{CCP}$ ) is regularly margined and will stay so. Hedges with 'smaller' NFC are regularly non-margined and will stay so as long as the counterparties stay small enough to dive under the clearing threshold.

But hedging with big NFC, which is fallen under the clearing obligation, will become stripped of an important option. In the days before EMIR, the Risk Triangle was fully available, the NFC under stress could have stopped margining. This door is now closed for an important segment of the market.

The big NFC are more important counterparties than the other two sets for at least two reasons: On the one hand, they might have an off-setting natural position, which forms the desired hedge for the hedging corporation. E.g., the power producer might find the needed carbon certificates exactly there and the big corporates might have an interest in hedging long term power supply. On the other hand, big NFC certainly understands the business of another big NFC and have sufficient deal expertise.

8. Reconsider your hedging activities

EMIR shrinks the set of realizable hedges considerably and this stands in contrast to the full hedge results from economic theory. In other words: On the one hand, you want to hedge as much as possible, on the other hand, the universe of ways of doing it is diminished by the regulator. EMIR might lead to making hedges a bit more exotic to exempt them of the clearing obligation - if done with the big corporates being under the clearing obligation-or building up significant coverage of middle-sized counterparties to get non-margined standard hedges in place.

39 For more details see http://www.handelsblatt.com/unternehmen/banken/kirch-vs-deutsche-bank-das-wohl-teuerste-fernsehinterviewaller-zeiten/6203632.html 
9. Reconsider your business model

Of course, there is a simple way out of the clearing obligation by dropping or shrinking the trading subsidiary. This runs counter to the maximization of shareholder value. Value generating business is cut down for regulatory reasons. This is as absurd as the forced move from big NFC as counterparties to smaller ones or the formation of a bank.

10. Do not create new risks through your liquidity buffer. In the outset, it has been mentioned that the regulatory developments in the U.S. and Europe lead to a significant increase in concentration risk. It is important not to create another concentration risk by holding only one class of bonds in your liquidity buffer. To illustrate take the example of a German corporation, which uses only German sovereign bonds for its liquidity buffer. Thereby it does not harvest the free lunch of diversification. For the sake of a tractable exposition, we consider the risk-optimal mix of bonds from only three issuers Germany (GER), Italy (ITA), and Spain (ESP) in Appendix B. Among all six risk-optimal strategies the global minimum variance portfolio based on Pearson's covariance is the most attractive alternativeand entails portfolio shares in Italian and Spanish bonds. If one wants to hold a liquidity buffer for a rolling one month period with little volatility in value and a small probability of loss, the global minimum variance portfolio is a good choice.

All things considered, it has got evident that the clearing obligation destroys a valuable (Triangle) option, impedes maximization of shareholder value, and puts the NFC in a needy situation without giving access to the lender of last resort. Maybe the ten recommendations can be of use in real-life treasury management.

As a final word, we add some Corona-specific advice, which has been summarized recently (Treasurer, 2020): First, there is as always the free lunch of diversification. Diversify your funding sources as one of them might dry out all of a sudden. This entails being ready to get an external rating as this is a precondition to tap the bond markets and diversify away from the loan market. Second, be aware of country risk which might lead to trapped cash. Third, make your operations resilient. This implies being ready for home office based value chains, which in turn needs proper digitalization of your company.

\section{References}

Basel Committee on Banking Supervision (2013). Basel III: The liquidity coverage ratio and liquidity risk monitoring tools. January.

Burger M, Graeber B, and Schindlmayr G (2007). Managing energy risk, first edition, Wiley \& Sons.

Danielsson (2011). Financial risk forecasting. First edition, Wiley \& Sons

de Socio A, (2011). The interbank market after the financial turmoil: squeezing liquidity in a "lemons market" or asking liquidity "on tap", Banca d'Italia Working Paper No. 819, September.

Ding, Y. and Schwert, A. (2010). Crisis period forecast evaluation of the DCC-GARCH Model, Honors thesis, Trinity College of Duke University

Embrechts, P., McNeil A.J. and Straumann, D. (1999). Correlation: Pitfalls and alternatives, Working Paper Departement Mathematik, ETH Zentrum, March.

Engle, R. (2000). Dynamic conditional correlation - A simple class of multivariate garch models. NBER Working Paper.

Engle, R. (2003a). Dynamic conditional correlation models of tail dependence, conference presentation on dependence modeling for credit portfolios.

Engle, R. (2003b). Risk and volatility: Econometric models and financial practice, Nobel Lecture, December.

Engle, R. and Manganelli, S. (2001). Value at risk models in finance, ECB Working Paper No. 75, August

E.ON. (2012). E.ON Debt Investor Update - Cleaner \& better energy, http://www.eon.com/de/investoren/anleihen/ praesentationen.html, December.

Grothe, O. Schnieders, J. and Segers, J. (2013). Measuring association and dependence between random vectors, preprint, Submitted for publication.

Heemann, M. and Delgado Rodriguez, P. (2013). US-Regulierung nach Dodd-Frank offenbart Unterschiede zu EURegeln, Borsenzeitung 39, 26 February.

Heston, S. (1993). A closed-form solution for options with stochastic volatility with applications to bond and currency options, Review of Financial Studies. 6. 328-343.

Heston, S.L. and Nandi, S. (1997). A closed-form garch option pricing model, Federal Reserve Bank of Atlanta.

Kartasheva, A. (2011). Metallgesellschaft AG and Its Hedging Program, Wharton MBA, Class of 2011, http:// anastasiakartashevaphd.com/mag-hp.pdf 
Kawai, M. and Zilcha, I. (1986). International trade with forward-futures markets, Journal of International Economics. 20, 83-98.

Kempf, A. Korn, O. and Sassning, S. (2011). Portfolio optimization using forward-looking information, CFR Working Paper NO. 11-10.

Korn, O., Kempf, A. and Sassning, S. (2011). A fully implied approach to find the global minimum variance portfolio, presentation at the 12th symposium on finance, banking, and insurance, December 15-16, Karlsruhe Institute of Technology, Germany.

Lehrbass, F. (2013). Counterparty credit risk and clearing of derivatives contracts, in: Credit Portfolio Securitizations and Derivatives, first edition, Wiley \& Sons.

Melo Mendes, B. and Leal, R. (2005). Robust multivariate modeling in finance. International Journal of Managerial Finance. 1(2), 95-107.

Merrill Lynch (1992). Merrill Lynch Bond Indices, Guide to the Merrill Lynch Global Index System. May.

Orskaug, E. (2009). Multivariate DCC-GARCH model - with various error distributions. Norwegian Computing Center, Thesis, June.

Osipovich, A. (2012). Uncleared margin rules threaten E\&P hedging, Energy Risk. November.

Treasurer Der (2020). Die fünf Corona-Lehren für das Treasury. Ausgabe. 17, September.

Pfaff, B. (2013). Financial risk modelling and portfolio optimization with R. First edition, Wiley \& Sons.

Yilmaz, T. (2010). Improving portfolio optimization by DCC And DECO GARCH: Evidence from Istanbul Stock Exchange, MPRA Working Paper, December.

\section{Appendix A - Econometric Details}

For the nine GARCH/APARCH models ${ }^{40}$ we get the following results in usual notation and ordered by the Akaike Information Criterion (AIC):

Table A1: Comparison of GARCH models

\begin{tabular}{|l|l|c|c|c|c|}
\hline GARCH(1,1) model & Conditional distribution & Log-likelihood & \#paras & Fixed para & AIC \\
\hline Heston Nandi & Normal & -9265 & 5 & & $-11,143$ \\
\hline GARCH & Student-t & -5157 & 4 & $-6,201$ & $-6,200$ \\
\hline APARCH & Student-t & -5157 & 5 & $\Delta=2$ & $-6,199$ \\
\hline GARCH & Skewed Student-t & -5157 & 5 & & $-6,199$ \\
\hline APARCH & Skewed Student-t & -5157 & 6 & $\Delta=2$ & $-6,192$ \\
\hline APARCH & Skewed Student-t & -5152 & 7 & & $-6,145$ \\
\hline GARCH & Normal & -5110 & 3 & & $-6,144$ \\
\hline APARCH & Normal & -5110 & 4 & & $-6,144$ \\
\hline APARCH & Normal & -5110 & 5 & & \\
\hline
\end{tabular}

An ad hoc approach would choose the model with the smallest AIC. ${ }^{41}$ Hence, the Heston Nandi GARCH would be the winner. But as the real test is the model's usefulness in real life, it would be a bit shortsighted to continue with just one model. Therefore we consider the second-best model in terms of AIC as well.

$40 \mathrm{APARCH}=$ Asymmetric Power ARCH. This model can handle not only fat tails but also the leverage effect, which GARCH models have a problem to cope with. We neglect the drift of daily log returns, which is empirically virtually zero.

41 If $k$ is number of parameters, $L L$ log-likelihood, and $n$ sample size the level of the Akaike Information Criterion is derived as: AIC $=(2 k-2 \mathrm{LL}) / n$. Note that this procedure is superior to just comparing likelihood levels. 


\section{Appendix A - Econometric Details (Cont.)}

Denoting the log-returns of the year ahead forward by $y_{t}$ we can specify the two selected models in more detail:

Heston Nandi GARCH (HN)

$\mathrm{y}_{\mathrm{t}}=\lambda \sigma_{\mathrm{t}}^{2}+\sigma_{\mathrm{t}} \mathrm{z}_{\mathrm{t}}$

with a time-varying variance

$\sigma_{\mathrm{t}}^{2}=\omega+\alpha\left(\mathrm{z}_{\mathrm{t}-1}-\gamma \sigma_{\mathrm{t}-1}\right)^{2}+\beta \sigma_{\mathrm{t}-1}^{2}$

where $z_{t}$ is iid standard normally distributed.

Student-t GARCH (ST)

$\mathrm{y}_{\mathrm{t}}=\sigma_{\mathrm{t}} \mathrm{z}_{\mathrm{t}}$

with a time-varying variance

$\sigma_{\mathrm{t}}^{2}=\omega+\alpha \mathrm{y}_{\mathrm{t}-1}^{2}+\beta \sigma_{\mathrm{t}-1}^{2}$

where $z_{t}$ is iid Student-t distributed.

Table A2: Comparison of parameter values

\begin{tabular}{l|c|c|}
\hline Parameter & HN & ST \\
\hline$\omega$ & 0.000000 & 0.000004 \\
\hline$\alpha$ & 0.000014 & 0.086176 \\
\hline$\beta$ & 0.900200 & 0.889475 \\
\hline$\gamma$ & 0.000000 & \\
\hline$\lambda$-Heston & 1.841000 & 6.727741 \\
\hline Shape & & 0.975650 \\
\hline Condition $(<1)$ & 0.900200 & \\
\hline
\end{tabular}

We give the estimated parameter values for the two models in the next table:

The last line indicates that the estimated parameter values of the two models fulfill the respective stationarity conditions. ${ }^{42}$ But there are differences in detail between both models.

The HN model has zero levels for two important parameters, which puts the model specification in question. Effectively a much simpler model seems to be favored instead of the full HN dynamics.

The essential dynamics for the conditional variance boil down to:

$$
\sigma_{\mathrm{t}}^{2}=\beta \sigma_{\mathrm{t}-1}^{2} \text {. }
$$

That the autoregressive term in the conditional variance is important is underlined by the estimated value for the same parameter in the ST model. The levels of 0.90 and 0.89 are close to each other.

In the HN model there is a high estimated level for the market price of risk (i.e., $\lambda$ ), which contradicts the initial unbiasedness assumption ${ }^{43}$ Nevertheless, we will use this high level later on in forecasting the $99 \%$ quantile of the log-returns.

The ST model surprises only in one place, i.e., with a small value for the first parameter. ${ }^{44}$ The "Shape" parameter in the last column is significantly below infinity and underlines that it is worthwhile not to work with a normal GARCH

42 For $\mathrm{HN}$ the last line shows the value of $\beta+\alpha \gamma^{2}$ and for ST the result of $\alpha+\beta$. Both values are below one.

43 Unbiasedness assumes a net-drift of zero. Hence, in the presence of big risk premia there should be a non-zero drift and thereby some bias.

44 We will see below that this parameter is highly significant. Hence, we do not force it to zero. 
Appendix A - Econometric Details (Cont.)

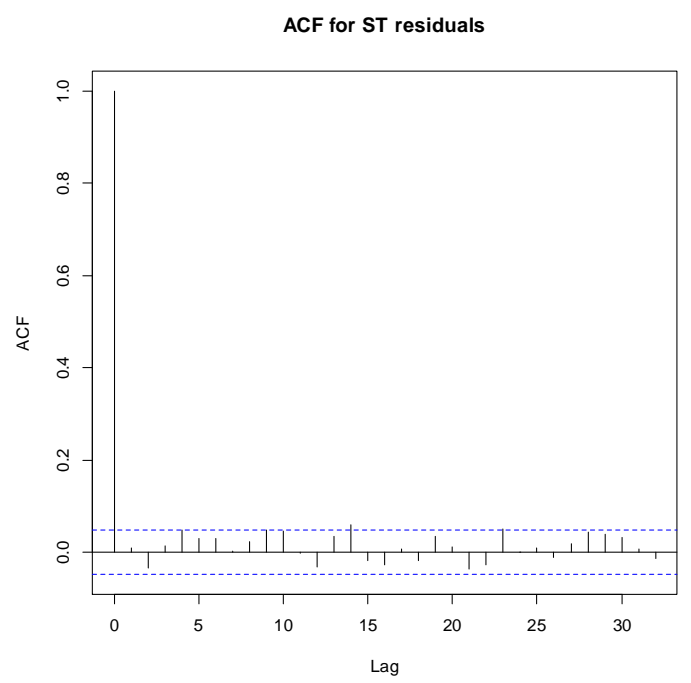

but a Student-t distribution. The reported level is in line with the literature: "In practice, the degrees of freedom are often estimated to be around 6 to 10" (Danielsson, 2011, 41).

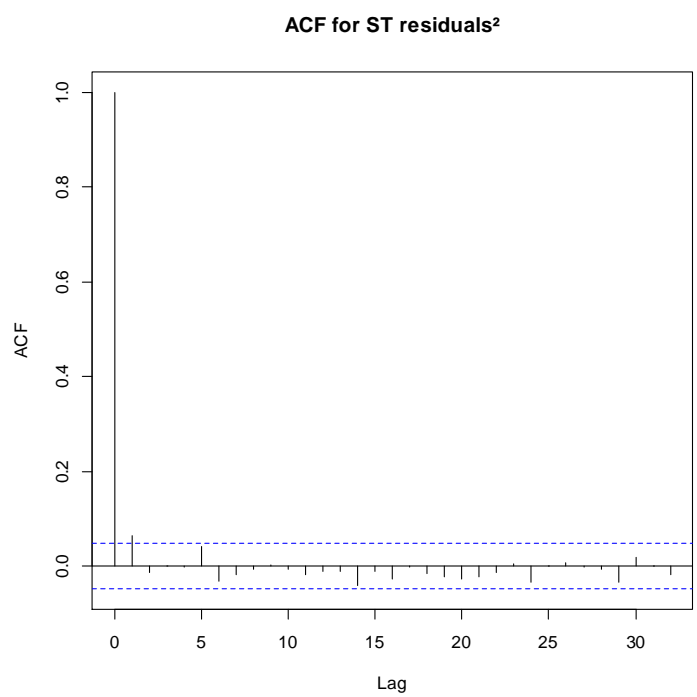

There are other arguments in favor of the ST model. We look at the relevant ACF. ${ }^{45}$

For the squared residuals the picture looks as follows.

The ACF of the residuals and squared residuals show that the ST model captures the essential structure in the residuals, i.e., nearly no significant autocorrelations are left.

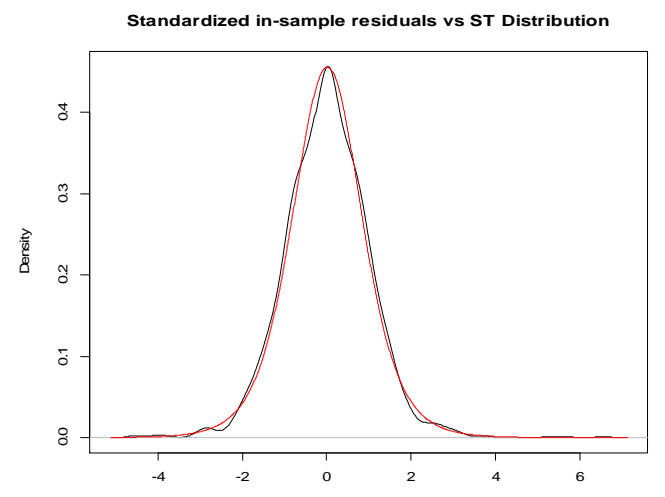

$45 \quad$ ACF $=$ Auto Correlation Function. 
Appendix A - Econometric Details (Cont.)

Now we plot the density of the residuals for the ST model.

The next chart plots theoretically expected quantile levels ( $\mathrm{x}$-axis) against empirical ones and is called a quantile to quantile plot (QQ Plot).

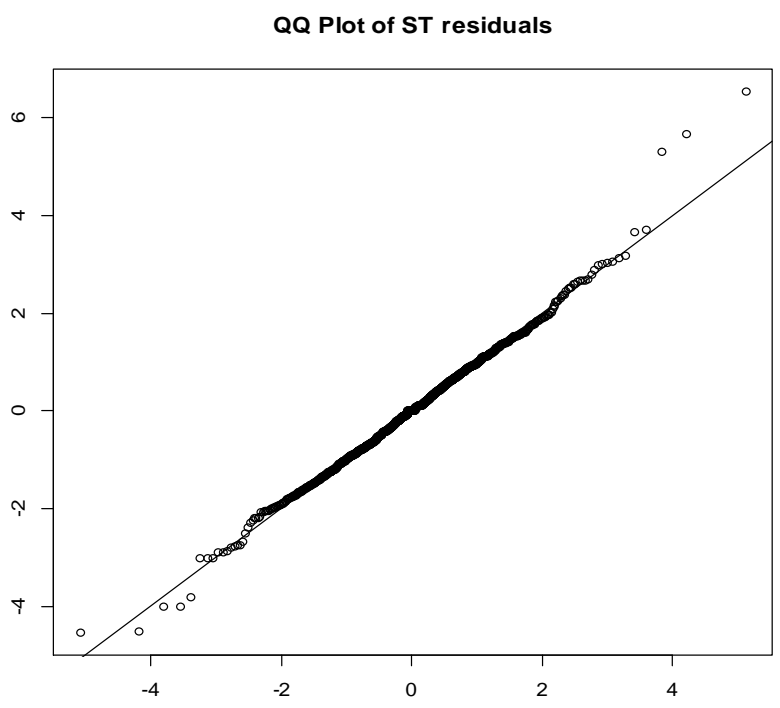

E.g. the single-digit percentage quantiles are mapped against each other in the lower-left corner of the chart. Let us start with the first observable quantile level, which is $1 / 1642$, i.e., 1 divided by sample size. The corresponding empirical quantile is the minimum value of the residuals amounting to -4.55 . If the residuals were ruled by the ST distribution the corresponding value would have been lower. This indicates a thin tail, but the points following to the right indicate the reverse, which is fat tails. This has been indicated by the preceding chart with the density already. What might not have been so clear is that the right tail is heavier than the left tail. This can be read from the QQ Plot by comparing the distances to the diagonal. Nevertheless, Ockham's razor made us prefer the simpler GARCH model without skew.

The maximum value of the residuals is 6.55 , which explains the y-axis endpoint.

\section{Appendix B - On the optimal mix of European sovereign debt within a liquidity buffer}

For the sake of a tractable exposition, we consider the risk-optimal mix of bonds from only three issuers Germany (GER), Italy (ITA), and Spain (ESP). Our exemplification can be spread to a wider universe of sovereign bonds if needed.

Speaking about risk we assume the perspective of an industrial corporate. This entails that the default risk of the sovereigns is out of scope. If e.g., Italy defaulted on their bonds as Greece did, the corporate certainly would have bigger problems due to the break-up of the Eurozone.

Roughly speaking a corporate treasurer, who seeks to form a liquidity buffer, which survives the Eurozone breakup, would try to achieve a target rating of better than the AA+ of the European Stability Mechanism (ESM). The ESM was established as a permanent firewall for the Eurozone to provide instant access to financial assistance programs for member states. As the ESM presumably would be wiped out by default of Italy, the rating quality of this scenario is evident.

Our perspective is also different from the well-known portfolio optimization, where expected return is maximized given some risk constrained. Here the constraint becomes the objective. More specifically we determine the portfolio weights, which minimize a given risk measure. Of course, we also report the mean return per strategy, but it is only of secondary interest.

We derive the optimal mix by applying six different approaches and compare the performance of each approach with the performance of holding German bonds only. Risk is measured by four indicators (5\% quantile, expected shortfall, standard deviation, and the probability to have a return below zero). 
Appendix B - On the optimal mix of European sovereign debt within a liquidity buffer (Cont.)

It will turn out that going for the "global minimum variance portfolio" based on Pearson's correlation is a promising approach, which is not outperformed by more modern approaches to estimate the dependence structure among the assets.

However, one promising modern approach could not be put into the competition, because it is impossible to get "implied correlations" on sovereign bonds. This is due to a lack of data. One should not forget that before CME's introduction of a "Sovys futures" in 2013 the alternatives to executing a sovereign yield spread are limited to combinations of cash government bonds and/or futures. Hence, we use bond data as summarized in the well-known Merril Lynch government bond indices to compare the six approaches.

\section{Description of and the first glance at data}

Analyzing fixed income yields is tricky if bonds are used with a remaining time to maturity of less than a year due to insufficient market liquidity. Hence, although the time horizon of the liquidity buffer is a month, it can be expected that only bonds with a remaining time to maturity of more than a year will be used in real-life management.

As corresponding data, we use the well-known Merril Lynch government bond indices ${ }^{46}$ for Germany (G0D0), Italy (G0I0), and Spain (G0E0) as in Grothe et al. (2013).

We point out some important properties of the indices as described in Merrill Lynch (1992). Each index is a market value-weighted total return index capturing all outstanding bonds of the issuing sovereign, which have at least one year time to maturity. The calculations are based on bid prices and the assumption that accrued interest and cash flows are reinvested daily (i.e., one day's worth of interest is treated regardless whether it is accrued or received as a coupon). Hence, total returns are based on the assumption that the index is bought and sold each day at the bid side of the market but without consideration of transaction costs. In light of the significant liquidity of the three markets, this assumption is not too restrictive.

Our data set starts with the factual introduction of the Euro in January 1999 and ends in October 2012. We use the period from Jan 2007 to the end to test the various approaches. This period is one of crises and contains much volatility and change in dependence structure. Therefore it appears rich enough for backtesting purposes.

For each of the six approaches, the portfolio weights are determined — using backward-looking information from a rolling window_-for the first time on 2 January 2007. The second time is on 30 January 2007, i.e., 20 trading days later and so on until the last rebalancing date on 26 September 2012. All in all, we have got 76 rebalancing dates, on which the portfolio weights are readjusted to their new optimal levels. This corresponds to a monthly adjustment of a liquidity buffer.

As the longest window under consideration is 750 days, we have to use data as early as Feb 2004 in our backtesting from Jan 2007 to Dec 2012. We call the period 2004 to 2012 "in-sample", whereas the latter period is called "backtesting period".

There is only one approach (i.e., the vector GARCH), which operates differently. Its parameters are estimated only once over the period January 1999 to December 2006. It then uses a covariance forecast valid for 2 January 2007 and so on, based on return information from the previous day only.

Table B1: In-sample statistics

\begin{tabular}{|l|c|c|c|}
\hline [Decimal] & GER & ITA & ESP \\
\hline Min. & -0.01156 & -0.03093 & -0.01928 \\
\hline 1st Qu. & -0.00129 & -0.00147 & -0.00162 \\
\hline Median & 0.00022 & 0.00019 & 0.00013 \\
\hline Mean & 0.00020 & 0.00017 & 0.00013 \\
\hline 3rd Qu. & 0.00168 & 0.00175 & 0.00174 \\
\hline Max. & 0.01651 & 0.03913 & 0.04600 \\
\hline Skewness & 0.03074 & 0.81301 & 1.40547 \\
\hline Excess Kurtosis & 1.94869 & 17.28103 & 16.98621 \\
\hline
\end{tabular}

46 The technical signifiers in brackets have to be read from left to right as follows: $G=$ Government, $0=$ all maturities above one year, $x=$ country code, $0=$ all quality. Data is from Bloomberg. 
Appendix B - On the optimal mix of European sovereign debt within a liquidity buffer (Cont.)

For the in-sample period the descriptive statistics of the data are as follows:

Boxplots of the returns show that they do not follow a normal distribution and exhibit typical features of financial time series.

Chart B1

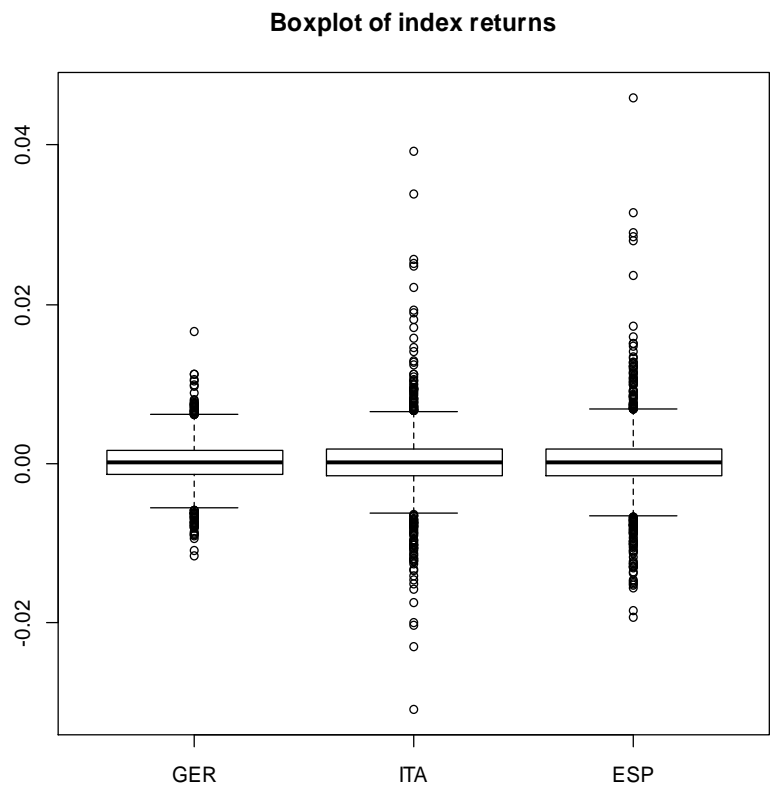

\section{Six approaches to determine risk-optimal portfolios}

Portfolio considerations crucially depend on understanding the dependencies between the returns from holding bonds from the three sovereign issuers. The dynamics of the dependencies have been described recently as follows: There is "a high association of north and south European government bonds at the beginning of [2007] ... and decreasing strength of association with the beginning of the year 2008. Economically this is to be expected since investors regarded the credit-worthiness of the countries in the European Union as homogeneous before 2008, which lead to similar yields on their government bonds. However, starting with the Euro-Crisis, investors started to question the rating of southern European countries ... and transferred their money to northern European countries" (Grothe et al., 2013).

The decreasing strength of association should be a tailwind for getting diversification into a liquidity buffer. Diversification can be achieved in various ways, which we sketch now.

\section{Global minimum variance portfolio (GMV)}

Markowitz's portfolio theory is known as a solution to the problem of selecting a portfolio from a set of risky assets when investors care only about mean and variance. But it is well-known that the "expected returns have proved hard to estimate ... Facing these implementation limitations, researchers are paying growing attention to the global minimum variance portfolio .... The only efficient portfolio that doesn't depend on expected returns" (Kempf et al., 2011).

Of course, there are alternative ways to derive the covariance matrix, which is used in the determination of the global minimum variance portfolio.

\section{GMVp - Global minimum variance portfolio based on Pearson's Covariance}

The simplest approach is based on Pearson's covariance of the returns of the bond indices, which is fine when you are coping with elliptical distributions like the normal distribution. But "unfortunately, most dependent real-world risks do not appear to have an elliptical distribution". In fact: "Correlation [or covariance] is simply a scalar measure of dependency; it cannot tell us everything we would like to know about the dependence structure of risks" (Embrechts et al., 1999). Therefore, to get a fuller picture other measures of dependency are used subsequently. 
Appendix B - On the optimal mix of European sovereign debt within a liquidity buffer (Cont.)

\section{GMVe - Global minimum variance portfolio based on Engle's Dynamic Conditional Correlation}

A more dynamic variant updates the covariance matrix by covariance forecasts from a Dynamic Conditional Correlation model (DCC) as introduced by Engle (2000). An example for this approach is found in Yilmaz (2010), who reports that "the results show that GMV portfolios constructed by DCC-GARCH outperformed the other portfolios". We motivate and introduce the DCC in short. In light of the well-known stylized facts of financial returns ${ }^{47}$ an ad hoc modeling choice would be a vector GARCH approach. But the curse of dimensionality makes us opt for a Dynamic Conditional Correlation (DCC) model. The three synchronized time series of daily returns of the three bond indices are denoted by $\varepsilon_{i, t}$ with $i=1,2,3$. The conditional correlation matrix of the standardized error terms is denoted by $R_{t}$. Denoting the conditional covariance of the pair $i, j$ of standardized error terms by $q_{i, j, t}$ allows to define the conditional covariance matrix.

The vector GARCH equations are:

$$
\begin{aligned}
& \varepsilon_{i, t}=h_{i, t}^{1 / 2} z_{i, t} \\
& h_{t}=\left(\begin{array}{l}
a_{1} \\
a_{2} \\
a_{3}
\end{array}\right)+\left(\begin{array}{l}
A_{11}, 0,0 \\
0, A_{22}, 0 \\
0, \quad 0, A_{33}
\end{array}\right)\left(\begin{array}{l}
\varepsilon_{1, t-1}^{2} \\
\varepsilon_{2, t-1}^{2} \\
\varepsilon_{3, t-1}^{2}
\end{array}\right)+\left(\begin{array}{c}
B_{11}, 0,0 \\
0, B_{22}, 0 \\
0, \quad 0, B_{33}
\end{array}\right)\left(\begin{array}{l}
h_{1, t-1} \\
h_{2, t-1} \\
h_{3, t-1}
\end{array}\right) \\
& z_{t} \sim N\left(0, P_{t}\right) \\
& P_{t}=D_{t} R_{t} D_{t} \\
& D_{t}=\operatorname{diag}\left(\sqrt{h_{i, t}}\right) \\
& Q_{t}{ }^{*-1}=\operatorname{diag}\left(1 / \sqrt{q_{i, j, t}}\right) \\
& \mathrm{R}_{\mathrm{t}}=\mathrm{Q}_{\mathrm{t}}{ }^{*-1} \mathrm{Q}_{\mathrm{t}} \mathrm{Q}_{\mathrm{t}}{ }^{*-1} \\
& \mathrm{Q}_{\mathrm{t}}=(1-\alpha-\beta) \overline{\mathrm{Q}}+\alpha \mathrm{z}_{\mathrm{t}-1} \mathrm{z}_{\mathrm{t}-1}^{\mathrm{A}}+\beta \mathrm{Q}_{\mathrm{t}-1}
\end{aligned}
$$

The covariance matrix $Q_{t}$ of the standardized error terms is dynamic and mean-reverts around the matrix of unconditional covariances, which is denoted by $\bar{Q}$.

Evidently, we are working with the diagonal specification of the DCC model to avoid an explosion in the numbers of parameters. Thus we have to estimate just nine parameters in (B2) and two more for (B8). With a full DCC we would have three matrices of dimension $3 \times 1,3 \times 3$, and $3 \times 3$, which would result in 21 parameters instead of 9 .

The two parameters in (B8) are indispensable as they drive the covariance matrix. That covariance is non-static is another well-accepted fact of financial data.

Nevertheless, the diagonal specification implies "that we do not have cross terms in the three GARCH models. Hence, only the own lagged variables are used in the dynamics" (Engle, 2003a). The model is estimated in two sequential steps: "The DCC method first estimates volatilities for each asset and computes the standardized residuals. It then estimates the covariances between these" (Engle, 2003a).

The dynamic nature of conditional variances and correlations makes the DCC model a non-linear combination of dependent normal variates. Hence, it is in vain to hope to get a normal distribution for the resulting portfolio returns.

Summing up the DCC model is parsimonious enough to get a promising relation between the number of parameters to be estimated and the in-sample size (i.e., $11: 2286=$ more than 200 sets per parameter) and can capture volatility and correlation clustering.

47 Fat tailedness, volatility clustering and the like. 
Appendix B - On the optimal mix of European sovereign debt within a liquidity buffer (Cont.)

\section{Estimation results}

Using the free software $\mathrm{R}$ we get:

Table B2: Parameter estimates for the DCC

\begin{tabular}{|l|c|c|c|c|}
\hline Estimate & GER $(\boldsymbol{i}=\mathbf{1})$ & ITA $(\boldsymbol{i}=\mathbf{2})$ & $\mathbf{E S P}(\boldsymbol{i}=\mathbf{3})$ & $\boldsymbol{Q}_{t}$ \\
\hline$a_{i i}$ & 0.00000006 & 0.00000005 & 0.00000005 & \\
\hline$A_{i i}$ & 0.03052940 & 0.03657661 & 0.02764284 & 0.03501334 \\
\hline$B_{i i}$ & 0.95563481 & 0.95290951 & 0.96121963 & 0.96347158 \\
\hline$\alpha$ & & & & \\
\hline$\beta$ & & & & \\
\hline Standard error & & & & \\
\hline$a_{i i}$ & 0.00000003 & 0.00906330 & 0.01413726 & \\
\hline$A_{i i}$ & 0.00000003 & 0.00874349 & 0.01308473 & 0.32681814 \\
\hline$B_{i i}$ & & & & 0.34962759 \\
\hline$\alpha$ & & & & \\
\hline$\beta$
\end{tabular}

The sum of each A and B is slightly below unity, which ensures stationarity of each individual GARCH. Being close to unity implies high persistence, which is good for our purposes as the convergence to the unconditional variance will be slow. This is a very important characteristic since it allows "to consider volatilities as quasistable, changing in the long run, but stable in the short period" (Engle and Manganelli, 2001). As often the GARCH parameter B has a bigger value than the ARCH parameter A. Hence, most of today's variance level flows into the conditional variance's level, which underlines the persistence. On the contrary the news about the volatility-as captured in the ARCH term-play a minor role.

While it is common knowledge how to use the individual GARCH models to forecast volatility one step ahead, this is less obvious for $Q t$, which we need when we want to consider dynamic covariances.

We apply the recursive algorithm as proposed by Ding and Schwert (2010) and as implied by (B8).

$\mathrm{q}_{\mathrm{i}, \mathrm{j}, \mathrm{t}}=\overline{\mathrm{Q}}_{\mathrm{i}, \mathrm{j}}(1-\alpha-\beta)+\alpha \mathrm{z}_{\mathrm{i}, \mathrm{t}-\mathrm{1}} \mathrm{z}_{\mathrm{j}, \mathrm{t}-1}+\beta \mathrm{q}_{\mathrm{i}, \mathrm{j}, \mathrm{t}-1}$

The ARMA-like equation (B9) highlights two consequences, which apply to covariance and correlation equally: "On average the correlations will be the same as in the data" (note the steady state) and "correlations rise when returns move together and fall when they move opposite" (Engle, 2003a), which can nicely be seen from the middle term on the right-hand side. As the sum of the two parameters is very close to unity, we may use the one day ahead forecast for the next twenty one trading days with a grain of salt. Thus we use the forecast on 2 January 2007 for the next rebalancing date on 30 January 2007, etc. A more exact approach can be found in Orskaug (2009).

\section{GMVs - Global minimum variance portfolio based on Spearman's Covariance}

The most well-known alternative to Pearson's correlation is Spearman's rank covariance, which is less sensitive against outliers. It should be noted that the Spearman matrix behaves rather like a correlation matrix with constant figures on the diagonal.

\section{GMVr - Global minimum variance portfolio based on Rousseeuw's Covariance}

A more recent approach makes use of a robustly estimated covariance matrix. Here we use the minimum covariance determinant (MCD) estimator of Rousseeuw, which is a covariance estimator which attains the maximum possible 
Appendix B - On the optimal mix of European sovereign debt within a liquidity buffer (Cont.)

breakdown point. "This measure is defined as the relative share of outliers in a sample such that the estimator does not take an arbitrary large value" (Pfaff, 2013,1p. 57).

The MCD approach has already been used in financial applications, e.g., Melo et al. (2005). There are many other variants of estimating the covariance matrix robustly, which are described in Pfaff (2013). To illustrate its merits we deem MCD to be sufficient.

\section{MTD - Minimum (lower) tail-dependent portfolio}

In contrast to the latter mentioned two approaches $(3 \& 4)$, there are approaches, which are fully focussed on the outliers. Going for the minimum (lower) tail-dependent portfolio is one example and coined as MTD.

We briefly give some background: Tail dependence is a conditional probability. It denotes the probability of observing a big (small) value for $X$, given a big (small) value for $Y$. It can be derived theoretically from an underlying dependence model such as a specific copula. Empirically upper and lower tail dependence functions can be estimated with respect to the empirical cumulative distribution $F$ and the indicator function 1[ ] as follows:

$$
\begin{aligned}
& L(u)=\frac{\sum_{k=1}^{\text {samplesize }} I\left[X_{k} \leq F_{X}^{-1}(u), Y_{k} \leq F_{Y}^{-1}(u)\right]}{\sum_{k=1}^{\text {samplesize }}\left[X_{k} \leq F_{X}^{-1}(u)\right]} \\
& U(u)=\frac{\sum_{k=1}^{\text {samplesize }}\left[X_{k}>F_{X}^{-1}(u), Y_{k}>F_{Y}^{-1}(u)\right]}{\sum_{k=1}^{\text {samplesize }}\left[X_{k}>F_{X}^{-1}(u)\right]}
\end{aligned}
$$

For the in-sample period we show a chart for the pairs of GER \& ITA and GER \& ESP, which shows L(u) and U(u) for varying u (see abcissa).

Chart B2:

GER\&ITA empirical tail dependence index vs Gauss Copula

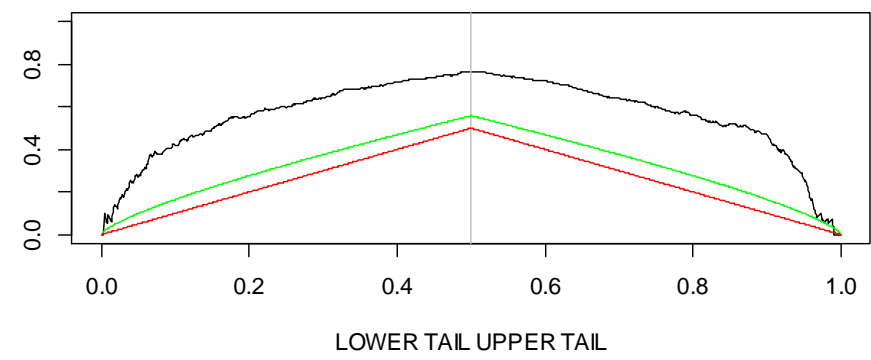

GER\&ESP empirical tail dependence index vs Gauss Copula

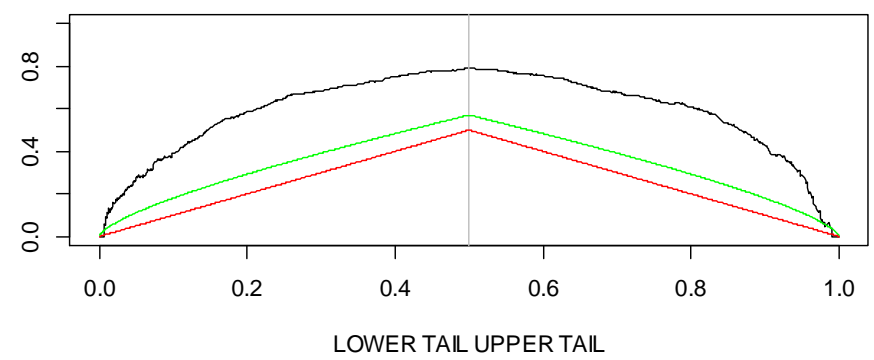


Appendix B - On the optimal mix of European sovereign debt within a liquidity buffer (Cont.)

The black line shows the empirical tail dependence. The green line is the theoretical level of a Gaussian copula if the empirical Pearson correlation is used. The red curve is from a Gaussian copula with zero correlation. Obviously both Gaussian curves approach zero if u moves to zero or one respectively as theory tells us.

For the empirical curve we have to dig deeper. Therefore we estimate two copulae with ML to get a feeling for the presence of tail dependence. The natural candidates are the Student $t$ copula (upper and lower tail dependence, i.e., in the limit on both sides a positive value is approached) and the Clayton copula (only lower tail dependence). It turns out that for both pairs the Student t copula reaches roughly four times the likelihood than the Clayton. Hence, the impression from the above charts — of having dependence in both tails—is not misleading.

Of special interest to real-world investors is the lower tail dependence, i.e., they do not care so much about the upper side. "Covariance/correlation takes deviations below and above the mean into account, but a risk-averse (long only) investor seeks positive returns ... and only considers negative returns (the downside) as risk. This point was made by Markowitz ... . In that sense, the lower tail dependence coefficient measures the strength of the relationship between asset returns when both are extremely negative at the same time" (Pfaff, 2013, p. 195).

Hence, it is natural to search for the minimum (lower) tail-dependent portfolio as an alternative to the global minimum variance portfolio. We call this approach MTD, where we make use of the R routine PTMD, which delivers the minimum lower tail dependent portfolio for a long only investor.

That this approach is rather heuristic gets evident from the following quote: "The function body is akin to that of the function PGMV by which the solution of a global minimum-variance portfolio is returned. But instead of employing the variance-covariance matrix as a measure of dispersion, the .. [lower tail dependence] matrix ... is utilized" (Pfaff, 2013, p. 200).

There are notable differences between the matrix of tail dependence and the covariance matrix. The latter is positive semi-definite by construction, whereas we can not be sure about the first to share this property. In fact, in empirical applications like ours, there might be time windows, where the matrix of lower tail dependence is not invertible, which we need to determine the global minimum variance portfolio.

\section{MES - Minimum expected shortfall portfolio}

There are various names for the mean loss above the Value at Risk: Conditional Value at Risk (CVaR), mean excess loss, tail VaR, and Expected Shortfall (ES). To find the MES portfolio we apply the R routine minriskPortfolio as described in Pfaff (2013, p. 238). We apply a confidence level of $95 \%$ for the VaR.

\section{Analysis of performance}

We derive the optimal mix by applying six different approaches and compare the performance of each approach with the performance of holding German bonds only. This portfolio is called GERo (= GERman bonds only). Having just three assets an additional consideration of a naïve diversification (i.e., 1/n rule") does not make sense and — by the way-leads to the worst results not shown here.

For the backtesting period the results of having invested EUR 1 on 2 January 2007 according to the six different strategies—with rebalancing every 20 trading days—are as follows:

\section{Chart B3: Wealth Indices based on number of rollovers $=76$}

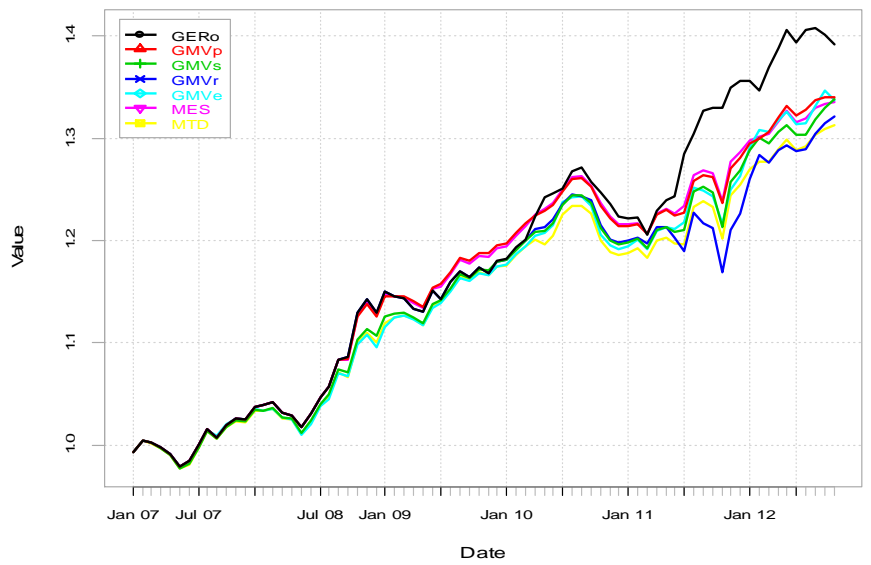


Appendix B - On the optimal mix of European sovereign debt within a liquidity buffer (Cont.)

Up to summer 2008 all strategies perform nearly indistinguishable, because "investors regarded the creditworthiness of the countries in the European Union as homogeneous" (Grothe et al., 2013). But then they depart from each other. Over the whole period, the biggest return is achieved by holding German bonds only.

But for the first phase up to 2010 MES and GMVp outperform GERo—although both strategies care for risk, whereas GERo does not take into account risk measures.

The only reason to prefer GERo would be the consideration of sovereign default risk, which is not in scope here.

In terms of relative performance GERo, GMVp, and MES appear most promising, although the dynamic GMVe, which is based on DCC, competes well with GMVp in the final phase. However, compared to the GMVs the outperformance is not that striking.

The two extreme strategies of looking only at outliers (MTD) or ignoring them (GMVr) rank last in terms of wealth.

Stopping the comparison here would be unfair as the strategies were developed to find risk-optimal portfolios. Hence, we list five risk measures in addition to the mean over the 76 holding periods:

Table B3: Mean return and risk measures (estimation with rolling window of 750 days)

\begin{tabular}{|l|c|c|c|c|c|c|c|}
\hline [Percent] & GERo & GMVp & GMVe & GMVs & GMVr & MES & MTD \\
\hline Mean & 0.442 & 0.391 & 0.389 & 0.377 & 0.389 & 0.364 & 0.387 \\
\hline StDev & 1.102 & 1.012 & 1.077 & 1.236 & 1.060 & 1.073 & 1.046 \\
\hline VaR & -1.102 & -1.058 & -1.070 & -1.130 & -1.129 & -1.063 & -1.080 \\
\hline ES & -1.208 & -1.439 & -1.851 & -1.951 & -1.780 & -1.853 & -1.484 \\
\hline ProbBelowZero & 39.474 & 34.211 & 35.526 & 40.789 & 38.158 & 38.158 & 35.526 \\
\hline
\end{tabular}

Comparing all risk-optimal strategies with each other the gobal minimum variance portfolio based on Pearson's covariance is the winner. It achieves the lowest probability to have a negative return, smallest ES and VaR and-by intention-standard deviation. Under the assumption of multivariate normal returns this would be no surprise. But as we have seen above the data is far from normal.

Taking the winner GMVp and comparing it to GERo we get a lower standard deviation, VaR, and probability to have a negative return. However, the ES is worse.

It certainly pays to check whether these results are stable with respect to shortening the estimation window from 750 to 250 days. The number of observations in the tail shrinks. This and numerical problems with MTD make us discount MTD from the following table.

Table B4: Mean return and risk measures (estimation with rolling window of 250 days)

\begin{tabular}{|l|c|c|c|c|c|c|}
\hline [Percent] & GERo & GMVp & GMVe & GMVs & GMVr & MES \\
\hline Mean & 0.442 & 0.371 & 0.414 & 0.386 & 0.379 & 0.358 \\
\hline StDev & 1.102 & 0.988 & 1.095 & 1.079 & 1.137 & 1.022 \\
\hline VaR & -1.102 & -1.102 & -1.068 & -1.079 & -1.252 & -1.072 \\
\hline ES & -1.208 & -1.401 & -1.921 & -1.671 & -2.211 & -1.446 \\
\hline ProbBelowZero & 39.474 & 32.895 & 36.842 & 34.211 & 31.579 & 34.211 \\
\hline
\end{tabular}


Appendix B - On the optimal mix of European sovereign debt within a liquidity buffer (Cont.)

There are some surprises: GMVr outperforms GMVp as concerns the loss probability, but the difference is too small to make us swap our favorite. GMVr is just one robust approach to the covariance matrix. Favoring GMVr should certainly be accompanied by testing all other robust approaches, which is out of scope here.

GMVe is slightly better as concerns the VaR. But weighing the complexity of DCC versus the ability to run GMVp with simple means makes us stick to GMVp. On top, the ES is still the lowest.

Hence, we conclude with a detailed look into the working of the GMVp strategy. First, we state that for both window sizes the mean weight of GER is around $60 \%$. We expect that with lowering correlations between GER and the other two indices their weights will increase. We plot a corresponding chart below for a window of 250 days to illustrate this:

Chart B4: Weights of Italian and Spanish bonds and correlations with German ones

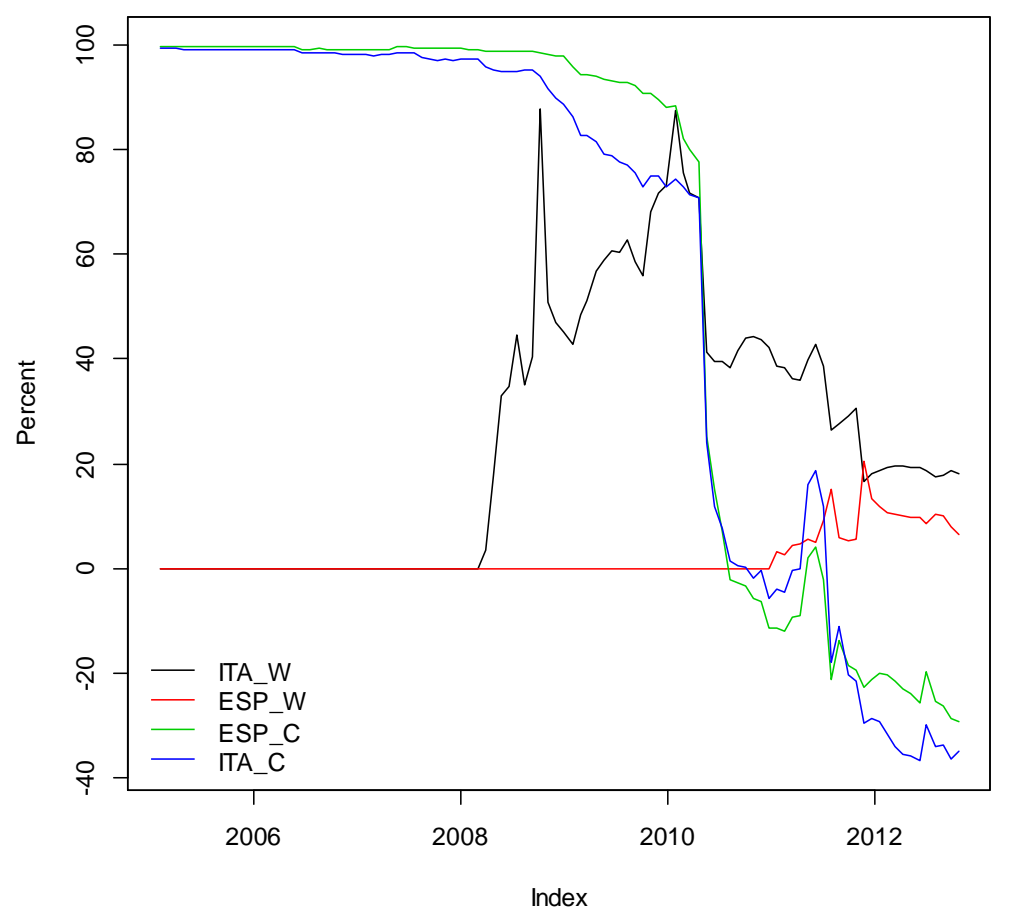

The correlations between the daily returns of the southern European indices and Germany's index are given in full percent and plotted in green and blue (ITA_C \& ESP_C). There has been a decrease already in 2007 because lower values of correlation in mid-2008 are based on 2007 data.

The weights for ITA and ESP (i.e., ITA_W \& ESP_W) behave differently. Already in 2008 Italian bonds reach a weight of up to $88 \%$, which gets lower in 2012. The holdings in Spanish bonds stay below $20 \%$ and move around $9 \%$ at the end. As soon as the correlations have entered negative territory holdings in both Southern European bond indices get interesting. This is an insight any portfolio manager would share without further debate.

Cite this article as: Frank Lehrbass (2021). Corporateliquidity risk management: Coping with Corona and theclearing obligation. International J ournal of M anagement Research and Economics. 1(1), 1-26. doi: 10.51483/ IJM RE.1.1.2021.1-26. 